Linköping Studies in Science and Technology

Dissertations, No. 1557

\title{
A Remote Monitoring and Control System for Cultural Heritage Buildings Utilizing Wireless Sensor Networks
}

Jingcheng Zhang

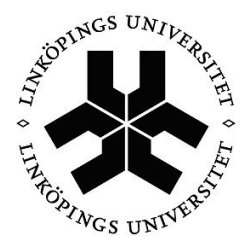

Linköpings universitet INSTITUTE OF TECHNOLOGY

Department of Science and Technology

Linköping University, SE-601 74 Norrköping, Sweden

Norrköping 2013 


\section{A Remote Monitoring and Control System for Cultural Heritage Buildings Utilizing Wireless Sensor Networks}

A dissertation submitted to ITN, Department of Science and Technology, Linköping University, for the degree of Doctor of Technology.

ISBN: 978-91-7519-448-6

ISSN: 0345-7524

Copyright $\odot$, 2013, Jingcheng Zhang, unless otherwise noted.

Linköping University

Department of Science and Technology

SE-601 74 Norrköping

Sweden

Printed by LiU-Tryck, Linköping 2013. 


\section{ABSTRACT}

This dissertation presents the study of a wireless remote monitoring and control system utilized for cultural heritage preservation purpose. The system uses wireless sensor networks to remotely monitor and control the indoor climate, i.e., temperature and relative humidity of the cultural buildings.

The system mainly consists of three parts, i.e., the wireless sensor network part, the gateway part and the web service part. Wireless sensor networks are deployed in different cultural buildings. The ZigBee protocol is utilized for the wireless sensor network communication. Sensor nodes report the indoor climate periodically. By connecting with radiators and/or dehumidifiers, the wireless control nodes can control the indoor climate according to the remote configuration. A gateway maintains the communication between a wireless sensor network and the web service. In monitoring function, the gateway forwards sensor messages from the wireless sensor network to the web service. In control function, the gateway synchronizes the climate settings from the web service to the wireless sensor network. The gateway also sends control commands to the wireless control nodes in the wireless sensor network. The web service provides a web-based user interface for the system.

Different from ordinary cable-connected sensor networks, a wireless sensor network that works for cultural heritage preservation should be a system with a large number of sensor nodes covering a large area in a building, high reliability in message transmission, low power consumption and low cost. In this study, the performance of the ZigBee wireless network is improved to meet such requirements base on the investigation of the ZigBee protocol limitation. Firstly, a method for enhancing the wireless sensor network communication reliability is developed. The reactive routing protocol defined by the ZigBee standard is improved so that the wireless nodes automatically detect and repair network communication problems. This method minimizes the message lost within the wireless sensor network by always reserving a route from the source node to the destination node. Secondly, a generic low power working method is developed for sensor devices. This method defines the general sensor module behavior which includes sensor data collecting, sensor message forwarding and wireless network rejoining upon communication failure. It allows 
sensor devices to maintain high message reliability with low power consumption. Especially, these methods are developed as a complementary infrastructure of the ZigBee wireless sensor network in order to increase the transmission reliability with low power consumption. Finally, methods and algorithms are developed to make it possible to power the ZigBee message relays (i.e., routers) with small batteries. In this system, the whole ZigBee network is synchronized. Wireless communications within the ZigBee network are scheduled so that every wireless transmission is collision-free. During the period when no communication is scheduled, the router can go into low power mode. This design improvement removes the original requirement of using mains power for ZigBee message relays. A truly battery-driven and low power consumption wireless sensor network is developed for monitoring cultural heritage buildings without (or with limited) mains power.

The remote control function is developed to mainly prevent biological degradation by controlling indoor climate, i.e., temperature and relative humidity. After studying the requirements for heritage preservation, a high flexibility, high reliability and low cost wireless indoor climate control system is developed. Different control algorithms are implemented to achieve different control results.

Till today, the remote monitoring and control system presented in this dissertation has been installed in 31 cultural heritage buildings both in Sweden and Norway. 


\section{POPULÄRVETENSKAPLIG SAMMANFATTNING}

Denna avhandling presenterar en studie och ett utvecklat system för trådlös fjärrövervakning och fjärrstyrning, med syftet att bevara kulturella och historiska byggnader. Systemet använder trådlöst sensornätverk för att övervaka och styra inomhusklimat, t.ex., temperatur och relativ luftfuktighet i byggnaderna.

Systemet består huvudsakligen av tre delar, nämligen trådlöst sensornätverk, gateway och webbtjänst. Trådlösa sensornätverk installeras i olika kulturella och historiska byggnader. ZigBee-protokollet utnyttjas för det trådlösa sensornätverkets kommunikation. Sensornoderna rapporterar periodiskt inomhusklimat. När element och/eller avfuktare är anslutna till trådlösa sensornätverket, kan de trådlösa styrnoderna styra inomhusklimat enligt konfiguration via webbtjänst. En gateway underhåller kommunikationen mellan trådlöst sensornätverk och webbtjänst. För övervakning skickar gateway vidare mätdata från ett trådlöst sensornätverk till webbtjänsten. För styrning, synkroniserar gateway klimatsinställningar från webbtjänsten till det trådlösa sensornätverket. Gateway-enheten skickar också styrkommandon till trådlösa styrnoder i trådlösa sensornätverket. Webbtjänsten är ett webb-baserat användargränssnitt för systemet.

I denna studie förbättras prestation av det standariserade ZigBee-protokollet baserad på undersökning av befintliga begränsningar. För det första, en metod utvecklas för att förhöja kommunikationspålitlighet av trådlösa sensornätverk, så att de trådlösa noderna automatiskt detekterar och reparerar kommunikationsproblem. För det andra, utvecklas en allmän lågeffektförbrukningsmetod för trådlösa sensornoderna, vilket tillåter sensorenheter att åstadkomma hög datakommunikationspålitlighet samtidigt låg effektförbrukning. Slutligen, har metoder och algoritmer utvecklats för att effektivt driva ZigBee-router på batteri istället för fast spänning. I detta system synkroniseras hela ZigBee-nätverket. Trådlösdatakommunikation i ZigBee-nätverket är schemalagd så att varje trådlös dataöverföring är utan kollision. Utanför schemalagda perioderna kan då ZigBee-routrar gå till lågeffektläge. Detta leder verkligen till ett batteri-drivet trådlöst sensornätverk med låg effektförbrukning, vilket passar bra för användning i de gamla kulturella och historiska byggnaderna. 
Populärvetenskaplig sammanfattning

Tills idag, har detta utvecklade system för fjärrövervakning och fjärrstyrning installerats i 31 byggnader i Sverige samt Norge. 


\section{ACKNOWLEDGEMENT}

First of all I would like to express my gratitude to my supervisor Professor Qin-Zhong Ye and Professor Shaofang Gong, for their guidance, supports and for giving me the opportunity to perform this challenging and interesting research work in the research group. Furthermore, I want to thank those people at the Department of Science and Technology who in various ways have supported me in my work. Especially, I want to express my gratitude to the following persons:

People in the Communication Electronics Research Group: Dr. Allan Huynh, Dr. Adriana Serban, Gustav Knutsson, Dr. Magnus Karlsson, and Patrik Huss.

Vinnova, Swedish Energy Agency, Swedish Church and Norrköping municipality are acknowledged for financial support of this work. Dr. Tor Broström, Jan Holmberg and Magnus Wessberg at Uppsala University are acknowledged for valuable inputs to the project.

Also thanks to all my dear friends for their friendship, all the laugh and support. They have really made these years a very pleasant journey.

Last but not least, I would like to thank my wife Juan Chen, my parents Shenghua Huang and Weijiang Zhang, my parents in law Suying Gu and Hongshuang Chen, for their constant support and love.

Jingcheng Zhang,

Norrköping, December 2013. 


\section{LIST OF PUBLICATIONS}

Papers included in this dissertation:

Paper 1 Jingcheng Zhang, Allan Huynh, Qin-Zhong Ye and Shaofang Gong, "Remote Sensing System for Cultural Buildings Utilizing ZigBee Technology", Proceedings of the $8^{\text {th }}$ International Conference on Computing, Communications and Control Technologies (CCCT 2010), Orlando, USA, pp. 71-77, April 2010.

Paper 2 Jingcheng Zhang, Allan Huynh, Qin-Zhong Ye and Shaofang Gong, "Design of the Remote Climate Control System for Cultural Buildings Utilizing ZigBee Technology", Sensors \& Transducers Journal (ISSN 1726-5479), Vol. 118, Issue 7, pp. 13-27, July 2010.

Paper 3 Jingcheng Zhang, Allan Huynh, Qin-Zhong Ye and Shaofang Gong, "Reliability and Latency Enhancements in a ZigBee Remote Sensing System", Proceedings of $4^{\text {th }}$ International Conference on Sensor Technologies and Applications (SENSORCOMM 2010), Venice/Mestre, Italy, pp. 196-202, July 2010.

Paper 4 Jingcheng Zhang, Allan Huynh, Qin-Zhong Ye and Shaofang Gong, "A Fully Wireless Monitoring and Control System for Protecting Cultural Heritage", Proceedings of $20^{\text {th }}$ IEEE International Conference on Collaboration Technologies and Infrastructures, Paris, France, pp. 51-57, June 2011.

Paper 5 Jingcheng Zhang, Allan Huynh, Qin-Zhong Ye and Shaofang Gong, "A Communication Reliability Enhancement Framework for the ZigBee Wireless Sensor Network", Sensors \& Transducers Journal (ISSN 1726-5479), Vol.135, Issue 12, pp. 42-56. 2012.

Paper 6 Jingcheng Zhang, Allan Huynh, Qin-Zhong Ye and Shaofang Gong, "A Web-based Remote Indoor Climate Control System Based on Wireless Sensor Network", International Journal of Sensors and Sensor Networks, Vol. 1, No. 3, June 2013.

Paper 7 Jingcheng Zhang, Qin-Zhong Ye, Allan Huynh and Shaofang Gong, "Design and Implementation of a Truly Battery-Driven ZigBee Wireless Sensor Network", Manuscript, September, 2013. 
The author has also been involved in the following papers not included in this thesis.

Paper 8 Allan Huynh, Jingcheng Zhang, Qin-Zhong Ye and Shaofang Gong, "ZigBee Radio with External Low-Noise Amplifier", Sensors \& Transducers Journal (ISSN 1726-5479), Vol. 114, Issue 3, pp. 184-191, March 2010.

Paper 9 Allan Huynh, Jingcheng Zhang, Qin-Zhong Ye and Shaofang Gong, "ZigBee Radio with External Power Amplifier and Low-Noise Amplifier", Sensors \& Transducers Journal (ISSN 1726-5479), Vol. 118, Issue 7, pp. 110121, July 2010.

Paper 10 Allan Huynh, Jingcheng Zhang, Qin-Zhong Ye and Shaofang Gong, "Wireless Remote System Monitoring for Cultural Heritage", Sensors \& Transducers Journal (ISSN 1726-5479), Vol. 118, Issue 7, pp. 1-12, July 2010. 



\section{LIST OF ABBREVIATIONS}

BER Bit Error Rate

CRC Cyclic Redundancy Check

CSMA-CA Carrier Sense Multiple Access - Collision Avoidance

dB Decibel

DHCP Dynamic Host Configuration Protocol

DUT Device Under Test

FFD Full Function Device

FTDI Future Technology Devices International

HART Highway Addressable Remote Transducer

HVAC Heating, Ventilation, and Air-Conditioning

IC Integrated Circuit

IEEE Institute of Electrical and Electronics Engineers

ITN Department of Science and Technology

IP Internet Protocol

kbps Kilo bits per second

LAN Local Area Network

LED Light Emitting Diode

LiU Linköping University

LNA Low-Noise Amplifier

LOS Line of Sight

LQI Link Quality Indication

MCU Microcontroller Unit

M2M Machine to machine

NWK Network

PA Power Amplifier

PCB Printed Circuit Board

PER Packet Error Rate

RF Radio Frequency

RFD Reduced Function Device

RSSI Received Signal Strength Indicator

Rx Receiver 
SC Synchronization Controller

SoC System-on-Chip

TI Texas Instruments

Tx Transmitter

UART Universal Asynchronous Receiver/Transmitter

UI User interface

USB Universal Serial Bus

UWB Ultra Wide Band

WLAN Wireless Local Area Network

WSN Wireless Sensor Network

ZC ZigBee Coordinator

ZED ZigBee End-Device

ZR ZigBee Router 


\section{Contents}

Abstract

Populärvetenskaplig sammanfattning ........................................................................ iii

Acknowledgement ............................................................................................................

List of Publications................................................................................................... vi

List of Abbreviations...................................................................................................... ix

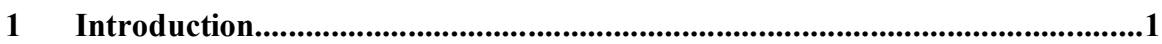

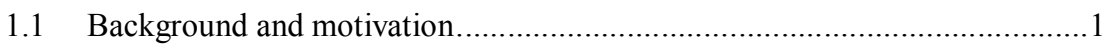

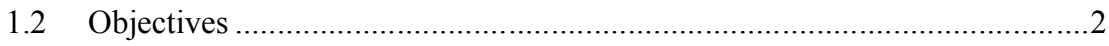

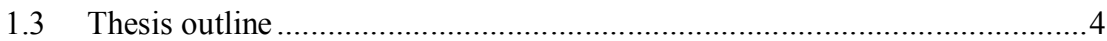

2 Applications and Protocols of Wireless Sensor Networks ...................................5

2.1 Introduction of three IEEE 802.15.4 based protocols ....................................

2.2 Protocol comparison.............................................................................

2.2.1 Protocol comparison according ISO 7-layer architecture ...........................7

2.2.2 Protocol comparison according to the cultural heritage preservation

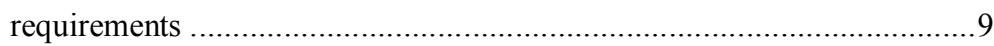

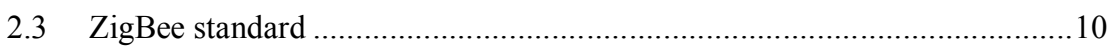

2.4 ZigBee WSN adaptation for heritage preservation purpose ..........................12

3 General System Description................................................................................13

3.1 Remote monitoring system architecture................................................... 14

3.2 Remote control function description....................................................... 14

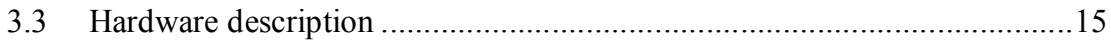

4 Remote Monitoring and Control System ......................................................19

4.1 Wireless monitoring and control networks..................................................19

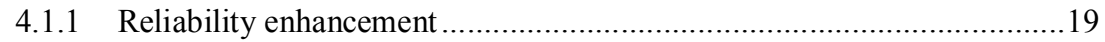

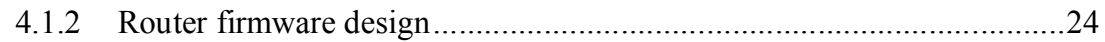

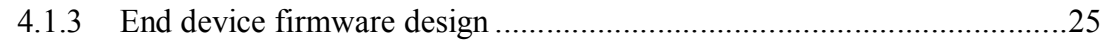

4.1.4 WSN control function Implementation.................................................. 31

4.1.5 Truly battery-driven ZigBee network ...................................................

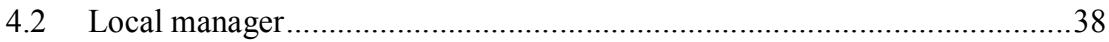

4.2.1 Local manager software architecture .....................................................39 


\section{Contents}

4.2.2 Monitoring and warning function .................................................. 40

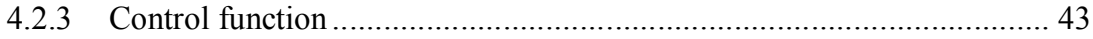

4.2.4 Control algorithm implementation.................................................. 47

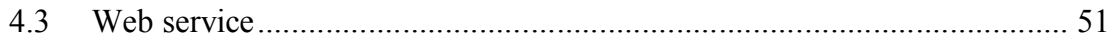

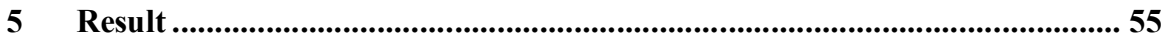

6 Summary of the Included Papers ....................................................................... 57

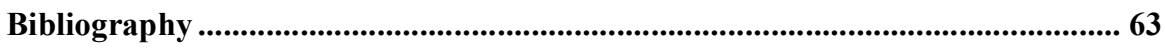

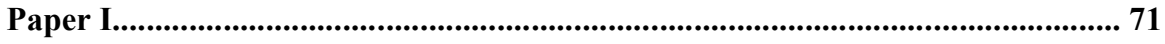

Remote Sensing System for Cultural Buildings Utilizing ZigBee Technology ...... 73

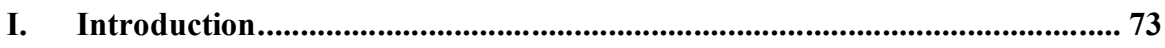

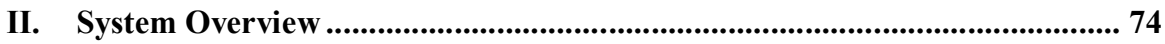

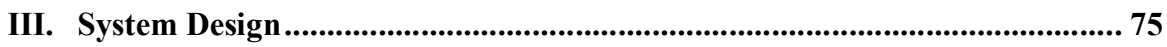

a. ZigBee network data collection package ............................................... 75

b. Local server data manipulation package................................................. 80

c. Main server web service package .......................................................... 81

IV. Test Setup .............................................................................................................. 82

a. Power consumption test set-up.......................................................... 82

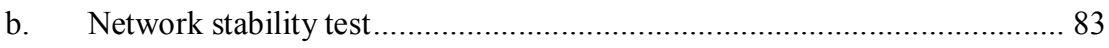

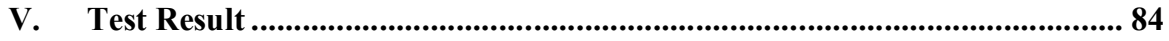

a. Current consumption test result ........................................................ 84

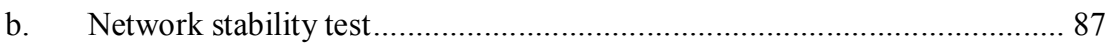

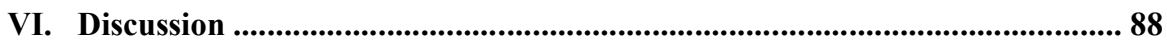

VII. Conclusion ................................................................................................................... 89

VIII.Acknowledgement ............................................................................................... 89

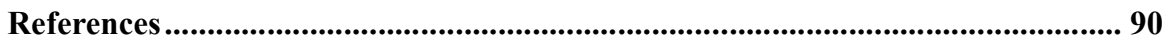

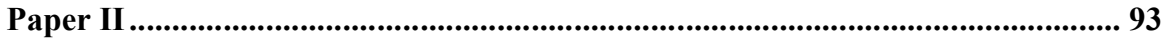

Design of the Remote Climate Control System for Cultural Buildings Utilizing ZigBee Technology ............................................................................................. 95

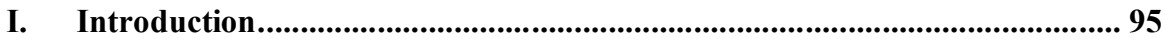

II. Remote Control System Overview ..................................................................... 96

III. Software Design of Wireless Sensor Network ................................................... 98 


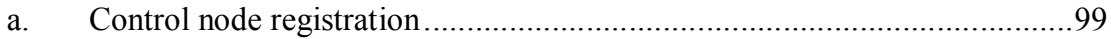

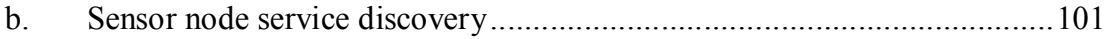

c. Sensor node working state machine ...................................................... 102

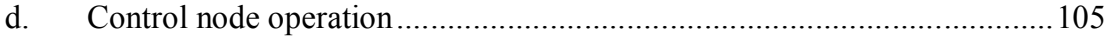

IV. Software Design of the Local Server ................................................................105
a. Control and sensor node registration....................................................... 106
b. Sensor reading information synchronization ............................................ 107
c. Local server command polling............................................................... 108

V. Software Design of the Main Server ...........................................................109

VI. Design Result Discussion............................................................................112

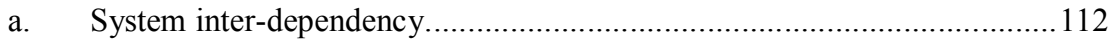

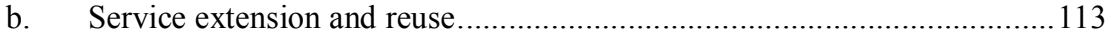

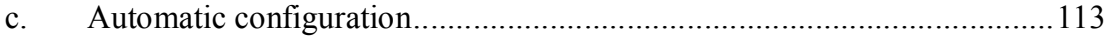

d. Passive command polling v.s. direct command forwarding ..................... 114

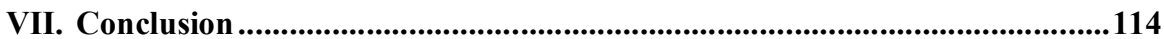

Acknowledgements.......................................................................................................114

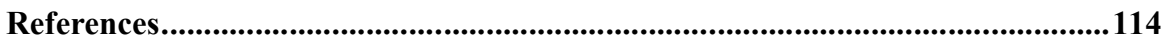

Paper III .........................................................................................................................117

Reliability and Latency Enhancements in a ZigBee Remote Sensing System......119

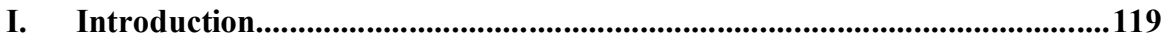

II. System Enhancement Software Design in ZigBee Network .........................121

a. Control and configure the network topology........................................ 122

b. Optimize the ZigBee network latency ................................................. 124

c. Routers restore network information from flash memory after power reset

III. System Enhancement Design in Local Server ..............................................126

a. ZigBee network failure warning function ............................................126

b. Software data buffer for Internet fault tolerance.....................................127

IV. Latency Test Set-up......................................................................129

a. AODV routing discovery minimum latency measurement ....................... 129

b. "Follow the topology" routing method latency measurement................... 131

V. Network Test Result..............................................................................................132 


\section{Contents}

a. Network latency test result summary

b. Temperature and humidity measurement results using our monitoring system

VI. Discussion

VII. Conclusion

Acknowledgement

References

Paper IV.

CultureBee - A Fully Wireless Monitoring and Control System for Protecting Cultural Heritage

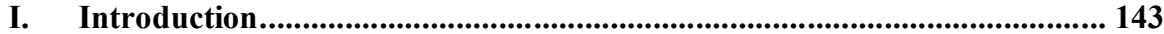

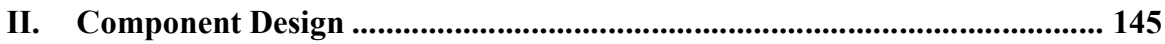
a. Wireless sensor network software design 145
b. Local server software design.
c. Main server software design

III. Wireless Sensor Network Optimization.................................................. 152
a. Wireless data forwarding method optimization .................................... 152
b. Connection status warning function .................................................. 155
c. System buffer function implementation .............................................. 157

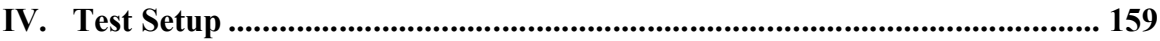

a. Power consumption test set-up........................................................... 159

b. AODV and "Direct parent forwarding" latency comparison.................... 160

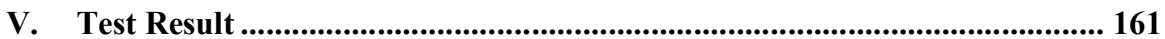

a. Power consumption test result .......................................................... 161

b. AODV and "Direct parent forwarding" performance comparison test result 162

c. CultureBee system deployment and measurement result ...................... 163

VI. Discussions ............................................................................................................... 166

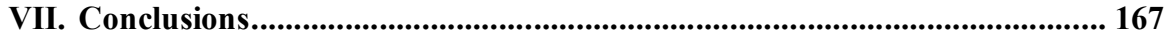

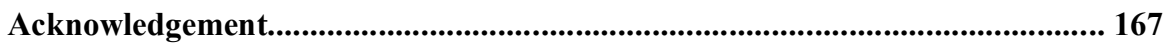

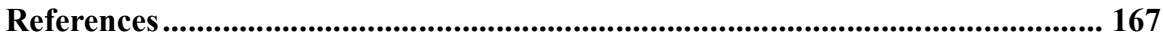

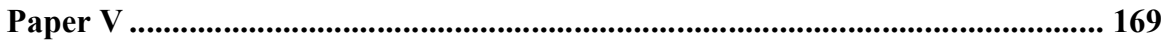


A Communication Reliability Enhancement Framework for Wireless Sensor Network Using the ZigBee Protocol ..........................................................171

I. Introduction.......................................................................................................171

a. End device cannot send message to the coordinator due to the router failure 172

b. New devices joining the sensor network by associating the router with broken link

c. Battery lifetime deceases when the end device lost connection with the sensor network

II. Message-flow Design to Increase the Network Communication Reliability 174

a. Add "handshake" messages between the router and the end device

b. Router with communication problem denies "association request" of the new device 175

c. End device detests the network before rejoin via association ...................176

d. Flash memory based circular buffer design for the end device 178

III. Design A General Purpose State Machine to increase the message reliability ...........................................................................178

a. End device reliability enhancement state machine …................................ 179

b. Router reliability enhancement state machine ........................................181

IV. Power consumption measurement............................................................183

a. Basic end device operation power consumption measurement ..................183

b. End device operation power consumption after reliability enhancement ...184

c. Battery lifetime calculation..................................................................... 187

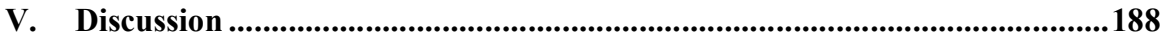

a. Utilize the framework to check the communication status other than the coordinator 188

b. Different architecture configuration of the enhancement framework with the

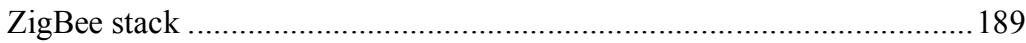

c. Possibility of lost sensor data under different interval configuration ......... 191

VI. Conclusion

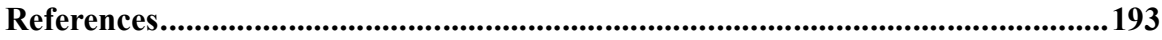

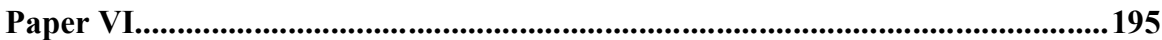

A Web-based Remote Indoor Climate Control System Based on Wireless Sensor Network 


\section{Contents}

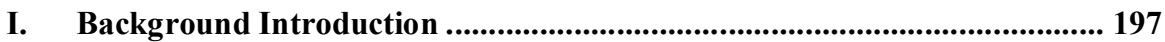

II. Remote Control System Architecture Description........................................... 198

III. Wireless Sensor Network .................................................................................... 200

a. Devices of wireless sensor network .................................................. 200

b. Wireless sensor network message flow .............................................. 202

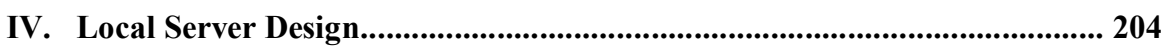

a. Control function component design ................................................. 206

b. Warning function component design..................................................... 210

V. Web Service Design ........................................................................................ 212

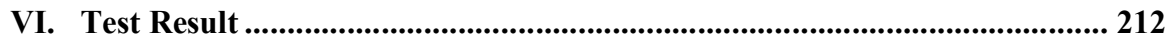

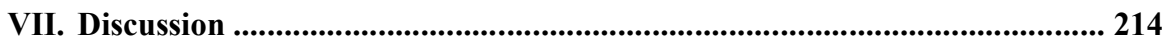

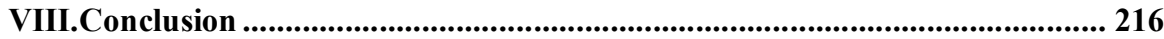

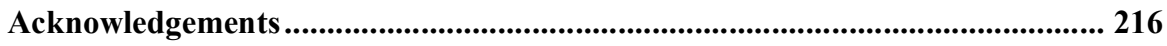

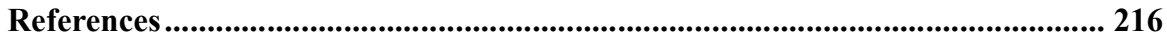

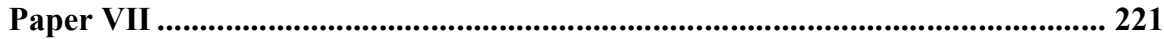

Design and Implementation of a Truly Battery-Driven Low Power ZigBee Wireless Sensor Network .............................................................................. 223

I. Background Introduction ................................................................................ 223

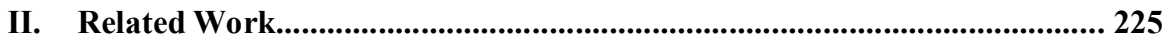

III. Wireless Sensor Network Device Hardware Construction .......................... 226

IV. ZigBee Router Synchronization Controller Software Design...................... 227

V. Communication Between Battery-Driven Router and the Concentrator ... 229
a. Router registration process
b. Uploading sensor message process .................................................. 231
c. Rejoining network process.................................................................. 233
d. Router operation state machine .............................................................. 234

VI. Communication Between Battery-Driven Router and End Devices............ 235
a. End device registration process.......................................................... 236
b. Sending sensor message process ..................................................... 237

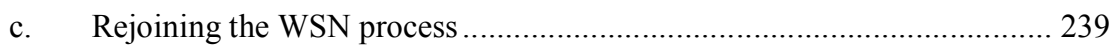

VII. Wireless Sensor Network Optimization............................................................. 242

a. $\quad$ Fetching sensor message from router .................................................. 242 
b. Sensor report time window allocation.......................................................24

VIII.Power Consumption Test Setup and Test Result...........................................246

a. Uploading sensor messages .............................................................247

b. Receiving sensor messages..............................................................251

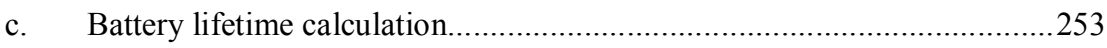

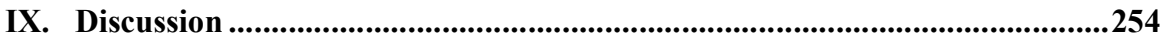

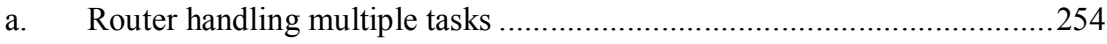

b. Time synchronization in the ZigBee WSN with battery-powered routers..255

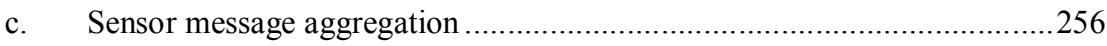

d. Battery-powered router WSN network scale ..........................................257

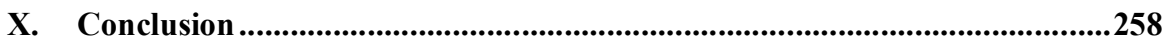

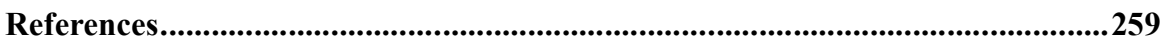





\section{Introduction}

Fast development of Information and Communications Technology (ICT) and emerging Internet of Things (IoT) systems bring many advantages to the modern society [1]. More and more sensor technologies are utilized in our daily life. By considering together with the collected ambient information, our life can become more efficient, more secure and more comfortable. The digital society based on massive data collection and smart data handling is the trend for the future development. Meanwhile, people are also looking for the possibilities to utilize wireless sensor technologies in cultural heritage preservation area. This dissertation presents a system solution of utilizing wireless sensor networks to remotely monitor and control the indoor climate of cultural and historical buildings for heritage preservation purpose.

\subsection{Background and motivation}

Cultural heritage preservation research based on mathematic modeling and material analysis requires long term and continues measurement results [2]. Each cultural building has its unique indoor climate characteristic. In order to generalize the common behavior, many buildings need to be measured at the same time. Among these buildings, some of them might be geographically located far away from each other. A web-based remote monitoring system can be used for data collecting purpose. By deploying data logging systems in different cultural buildings, the measured data can be forwarded to the remote web service via the Internet. Such data can be further analyzed according to specific research requirements. 


\section{Objectives}

Cultural heritage buildings, e.g., churches or museums, should also provide a good indoor environment for human activities. During this time, the indoor climate of the cultural building should be adjusted to a comfortable level. When the building is not used, the indoor climate should be maintained for heritage preservation purpose. Biological degradation problems, e.g., mold, should be avoided in the cultural heritage buildings. A remote control function can be developed for both requirements. Users could remotely control the indoor climate in cultural buildings by issuing activity schedules from the web service. During the time when the building is not used, the control system maintains the minimum climate requirement for heritage preservation purpose.

\subsection{Objectives}

Wireless sensor networks can bring lots of benefit for cultural heritage preservation. Firstly, compared with the wired system, a wireless system installation requires much less building modifications. Wired communications are replaced by wireless communications. By installing such systems, the cultural heritage buildings are maximally kept unchanged. Secondly, compared with wired system, the wireless system deployment is much more cost effective. The wired system roughly cost $\$ 10$ per meter which including the material fee and installation cost [3]. In contrast, a wireless device can be deployed anywhere within the wireless communication range. Thirdly, wireless systems are much more flexible and easy to be extended. By utilizing wireless communications, wireless sensor nodes can be placed at anyplace within the wireless network range. Many measurement points can be easily added according to the requirements. For example, a wireless sensor for measuring temperature and relative humidity can be put into the wardrobe for mold problem detection. After the measurement, the same sensor could be moved to another place for other microclimate detection purpose.

However, implementing a wireless sensor network for remote monitoring of cultural heritage buildings is very challenging. Different from a normal living environment, cultural heritage buildings have some unique characteristics. Firstly, these types of buildings are usually much bigger than ordinary houses. They were usually constructed from stone and built in many floors. In order to measure the indoor climate in such buildings, around 20 to 40 sampling points are required for each building. For each 
point, the measured result should be reported every 15 minutes. Secondly, most of the cultural buildings are built before the invention of electricity. Power outlets in such buildings are very limited. Thirdly, the media inside the cultural buildings can change dynamically. For example, the relative humidity can change from 20 to $95 \%$ from winter to summer. The indoor climate fluctuation brings a lot of uncertainty to the wireless signal transmission [4]. Nevertheless, as a monitoring system, the package loss rate [5] should be as low as possible. By summarizing the requirements, a wireless sensor network that works for cultural heritage preservation should have the following characteristics.

- Large scale wireless sensor network: The size of the wireless sensor network should be large enough to cover a big cultural building.

- Low power consumption: Power outlets are very limited in cultural buildings. The wireless sensor network should be mainly powered by battery with long battery lifetime.

- High message transmission reliability: The measured data should be successfully sent from the wireless sensor network to the web service.

Different from the monitoring system, the remote control function focuses on preserving cultural buildings which are "occasionally used". Usually, a daily used church is well equipped with heating and ventilation systems. Such systems can provide good environment for both human activities and heritage preservation. However, situations in the "occasionally used" churches are different [6]. Firstly, such churches are only used several times per year. It is neither practical nor effective to maintain a similar indoor climate as daily used churches. Secondly, the size of such churches is usually small. Heating systems in such churches are very old and low efficient. Thirdly, due to lack of maintenance, the relative humidity inside the church can be higher than $80 \%$. In order to preserve heritage in such churches, a low cost, easy-to-install and maintenance-free indoor climate control solution is highly appreciated. Especially, the remote control function should provide the following features.

- A web based control system which can remotely configure the indoor climate in different cultural buildings.

- Two kinds of control mode. One mode works for providing a good indoor climate during human activities. Another mode works only for heritage 


\section{Objectives}

preservation when the building is not used. Compared with the temperature required for human activities, the temperature requirement for heritage preservation is always lower [7]. A lot of energy can be saved by maintaining a lower temperature, which is good for small churches with limited budget.

- Different control algorithms. Different control algorithms should be developed for different control purposes. During the human activities, the indoor temperature should be maintained in a comfortable level. During the time when the church is not used, the relative humidity should be controlled to prevent mold problem.

- High flexibility. The same control node could work for different control tasks based on the user configuration. For example, a wireless control node can be configured to control temperature by connecting a radiator. Afterwards, the same control node could also be configured to control relative humidity by connecting a dehumidifier.

- High reliability. All control operations should be done under surveillance.

\subsection{Thesis outline}

This thesis is organized as the following. Chapter 2 describes the existing wireless sensor network standard. The ZigBee standard is selected as the communication protocol for the wireless sensor network. By comparing the ZigBee protocol with the cultural heritage preservation requirements, limitations of the ZigBee protocol are listed. Chapter 3 presents the system architecture of the remote monitoring and control system. It also includes the description of hardware devices utilized in this study. Chapter 4 elaborates the design and implementation for the remote monitoring and control functions. The wireless sensor network, the gateway and the web service are presented accordingly. Chapter 5 includes a summary of the study results. 


\section{Applications and Protocols of Wireless Sensor Networks}

A wireless sensor networks (WSN) usually consists of wireless devices installed with radio transceivers. Distributed mechanism is utilized in the WSN so that an ad-hoc WSN topology is created which can be changed dynamically. WSN was first motivated by military monitoring applications during the 1980s. Till today, large scale WSN applications are becoming a reality, for example, Smart Grid [8] [9] [10] [11], internet of things [12] [13] [14] and Machine to machine (M2M) communication networks [15] [16] [17]. The idea of utilizing wireless technology on cultural heritage preservation purpose has been proposed for many years. Lots of researches have been done on monitoring the structural health of a building by detecting building vibration [18]-[25] Utilizing wireless sensor network for indoor climate monitoring and control is also an active research area [26]-[29]. Different applications on utilizing wireless sensor network are presented in different novel methods.

Besides different applications, the development of WSN infrastructure is always aiming at low-power and low cost. As the network scale is growing bigger and bigger, power efficient routing algorithms for large scale WSN are more and more crucial for the network performance. To achieve this goal, a common approach is to divide a large WSN into different smaller zones using topology control methods. J. N. Ai-Karaki et al. proposed a data power-efficient routing paradigm using data aggregation [30]. Wireless devices are virtually divided in different zones. Inside each zone, a wireless node is optimally selected as the master. Sensor data are collected in two levels, both locally (inside the zone) and globally (inter-zone). This method assumes that all wireless devices are extremely stationary since the zone reestablishment introduce very high overhead into the WSN. Q. Li et al. proposed a hierarchical power-aware routing 
algorithm [31]. A global zone to zone path is calculated according to the power level of each zone. The path is controlled by a global controller which could be the wireless node with the highest power. Alternatively, a multi-zoned wireless sensor network could have more than one data sink. F. Ye et al. proposed a so called Two-Tier Data Dissemination (TTDD) [32] algorithm which delivers sensor messages to multiple mobile data sinks. The routing paths between the source and the data sink are actively discovered by the sensor using greedy geographical forwarding [33]. TTDD algorithm provides an efficient message routing by assuming that a very accurate positioning system is equipped in the WSN.

Moreover, many standards and protocols are defined for WSNs [34]. Generally, these protocols can be categorized as Media Access Control (MAC) protocols and network protocols. MAC protocols [35] [36] [37] [38] mainly focus on message transmission, throughput efficiency and power management. Network protocols [39] [40] [41] mainly designed for topology control, ad-hoc and mesh features and resource management. Among many WSN protocols, one of the most important MAC protocols is IEEE 802.15.4 standard. As first released in 2003, the IEEE 802.15.4 standard is defined aiming at providing a fundamental low level network link communications for wireless personal area network (WPAN). The focus of this standard is low-cost and low-speed communication between devices. As the major network protocols based on IEEE 802.15.4 standard, ZigBee, WirelessHART [42] and ISA100.11a [43] protocols are introduced in this chapter. These three protocols are compared according to the ISO 7 layer architecture. Eventually, ZigBee standard is selected as the communication protocol for wireless sensor networks according to the cultural heritage preservation requirements. Eventually, the limitations of the ZigBee standard are listed at the end of this chapter.

\subsection{Introduction of three IEEE 802.15.4 based protocols}

The ZigBee protocol is the first global standard for wireless sensor network. A typical ZigBee network consists of end devices, routers and one coordinator. The end device can be set to low powered mode so that it can be powered by battery. Routers and the coordinator are also called mesh nodes which works together to provide mesh feature of the ZigBee network. As a result, routers and the coordinator cannot go to low power mode and are usually mains powered. In the network layer, the ZigBee protocol utilizes 
reactive Ad hoc On-Demand Distance Vector (AODV) [44] routing algorithm. The application area of ZigBee protocol is very large, by defining different application profiles, ZigBee devices from different manufactures can inter-communicate with each other.

WirelessHART protocol is developed aiming at replacing the cable of HART protocol [42] in industry process control. Different from the ZigBee protocol, a WirelessHART network is a flat network. All wireless nodes in the wireless network play the same role. WirelessHART communications are precisely scheduled based on Time Division Multiple Access (TDMA) [45] and employ a channel-hopping scheme for added system data bandwidth and robustness. Graph routing [46] and source routing methods are applied in the WirelessHART network. Additionally, a network manager device is always introduced in the WirelessHART network which continuously adapts the network graphs and network schedules to changes in the network topology and communication demand [47] [48].

ISA100.11a standard is developed aiming at supporting a wide range of wireless industrial plant needs, including process automation, factory automation and RFID. The network and transport layers are based on 6LoWPAN [49] [50], IPv6 and UDP standards [51]. Instead of the standard Media Access Control (MAC) layer [x], the graph routing, frequency-hopping and time-slotted time domain multiple access features are implemented in the MAC layer. The ISA100.11a protocol provides an open platform for different applications. The protocol only specified the interface for the network manager in the application layer.

\subsection{Protocol comparison}

\subsubsection{Protocol comparison according ISO 7-layer architecture}

A detailed comparison of ZigBee, WirelessHART and ISA100.11a protocols are shown in this part. The comparison adopts ISO 7-layer [52] concept, the comparison result is presented according to different layers. 


\subsubsection{Physical layer}

All three protocols utilize the physical layer defined by the IEEE 802.15.4-2006 2.4GHz DSSS [55] physical layer.

\subsubsection{Data link layer}

The ZigBee protocol utilizes the non-beacon enabled MAC layer defined by IEEE 802.15.4. Full-function device (FFD) and Reduced-function device (RFD) are further defined as mesh node and end device accordingly. The start topology is directly adopted from IEEE 802.15.4 standard. The multi-hop network and mesh features are implement by upper layers.

The WirelessHART protocol data link layer fully complaints the IEEE 802.15.4 MAC layer. Additionally, a $10 \mathrm{~ms}$ timeslot, a synchronized frequency hopping and the time division multiple access are included in order to provide a collision-free and deterministic communications. In the frequency hopping mechanism, the WirelessHART protocol also defined a blacklisting function which prevents the frequency hopping to the channel with interference.

Similar as WirelessHART, the ISA100.11a standard also implements the time slot, frequency hopping mechanism in the MAC layer. Although configured as $10 \mathrm{~ms}$ by default, the timeslot interval is configurable. Moreover, the graph routing features are also implemented in this layer.

\subsubsection{Network layer}

The network layer of these three protocols maintains the network related information, e.g., routing tables, neighbor tables. When routing big data, the routing algorithms utilized in these three protocols also support message fragmentation. Although different routing algorithms are applied, all of them provide end-to-end routing functions and high security.

In ZigBee protocol, the AODV routing algorithm is applied. The routing records are created on demand. The routing record creation introduces big network overhead due to 8 
the message broadcasting. The WirelessHART network layer provides both source routing and graph routing functions. Multiple preconfigured routes to different destinations are prepared in each device. Such routing method provides high message transmission reliability and time efficiency by sacrificing hardware resource in the wireless devices. The ISA100.11a standard utilizes IPv6 and 6LowPAN routing protocol in the network layer. A routing-over and mesh-under routing method is implemented.

\subsubsection{Application layer}

As a wireless standard for resource restricted embedded device, the transport, session and presentation layers are undefined in all three standards. Different wireless sensor network applications are defined in the application layer. All three protocols define the network management interfaces in the application layer.

Specifically, the ZigBee protocol defines different profiles for different application areas. Wireless sensor network applications can be developed by using standard interfaces. A generic service discovery mechanism is defined in the ZigBee protocol which allows different ZigBee wireless nodes discover the feature characteristics of each other. The HART command-line based interfaces are defined by the WirelessHART protocol in the application layer. Different from WirelessHART protocol, the ISA100.11a standard is not strictly bound with industry process control. Users are free to define different applications in the application layer of ISA100.11a standard.

\subsubsection{Protocol comparison according to the cultural heritage preservation requirements}

Compared with ZigBee protocol, WirelessHART and ISA100.11a protocol are developed aiming at wireless industry control. The frequency hopping, synchronized time slot and graph-routing based mesh network mechanism implemented in these two protocols can provide much better performance compared with ZigBee protocol. However, this study is to select the WSN protocol which most suitable for cultural heritage preservation rather than the one with best performance. 
First of all, compared with the industry environment, the media in the cultural heritage buildings are usually much less noisy. The chance for a wireless signal to be interfered is much less than in the industry environment. Secondly, the monitoring result is not as time-critical as required in industry process control. The reactive AODV routing algorithm is usually good enough for temperature and relative humidity monitoring purpose. The measurement points in the cultural heritage building are stationary in most of the time. The resource consuming routing algorithm does not need to be utilized all the time. Finally, by applying a relatively simpler routing algorithm, the hardware requirement for ZigBee wireless device is lower than the WirelessHART device and ISA100.11a device. In other words, the ZigBee wireless sensor network can be more cost-effective. Base on these reasons, the ZigBee protocol is selected as the communication protocol of the wireless sensor network for cultural heritage preservation purpose.

\section{$2.3 \quad$ ZigBee standard}

The ZigBee standard is the first global standard aiming at low power, low complexity and low cost wireless sensor networks. The ZigBee standard can be utilized to create a self-organized, self-healing and multi-hop wireless sensor networks. The protocol architecture [53] is shown in Figure 1.

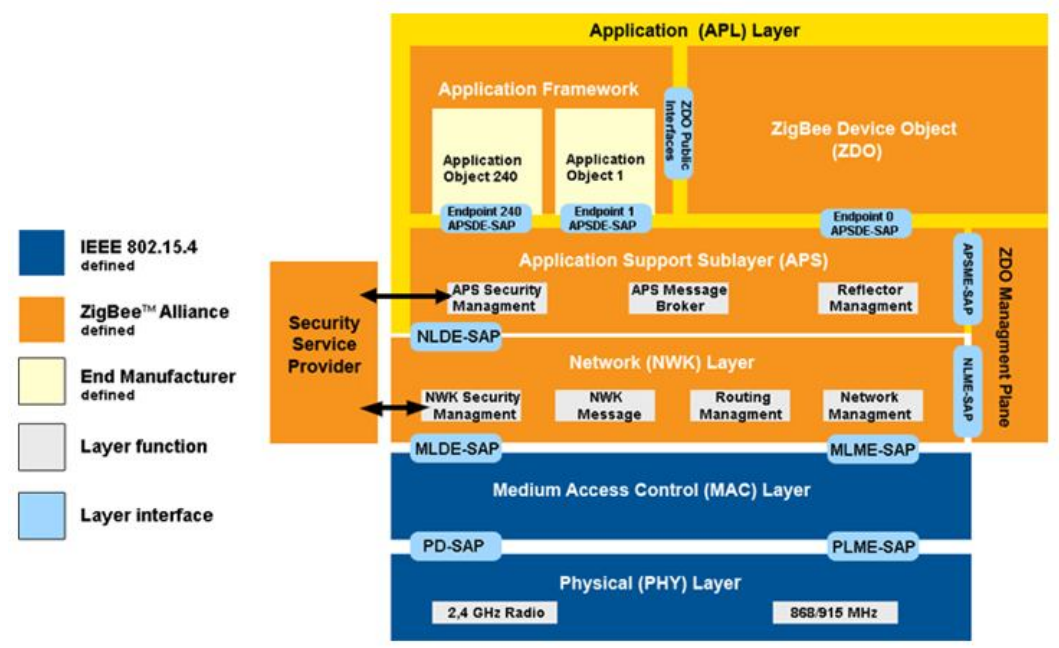

Figure 1. ZigBee software protocol architecture [53]. 
As shown in Figure 1, the ZigBee protocol architecture contains four layers. The Physical layer (PHY) and the Medium Access Control (MAC) layer are defined by the IEEE 802.15.4 standard [55]. It includes the hardware specification and the radio transmission specification. The network and application layers are defined by the ZigBee standard. The mesh features maintained by the network layer enables the selforganization and self-healing functions of the ZigBee network. The application layer mainly consists of three components: the Application Support Sub-Layer (APS), the ZigBee Device Object (ZDO) and the Application Framework (AF). The wireless sensor network applications are also defined in this layer. Different applications could contain application profiles [56] which define standard communication interfaces between products from different manufactures.

A typical ZigBee network consists of end devices (ZED), routers (ZR) and a coordinator $(\mathrm{ZC})$. The router and coordinator are also called mesh nodes which maintain mesh features and routing functions for a ZigBee network. The only difference between router and coordinator is that the coordinator is the creator of one ZigBee network. Both a router and a coordinator can be assigned as the concentrator. A concentrator is considered as the final destination of all application related messages. The route to the concentrator is optimized by many-to-one routing method [57]. The end device does not participate any message routing. When an end device wants to send any message, it always forwards the message to its parent device, which could be a router or the coordinator. The parent device helps the end device to forward the message to the destination. Since an end device does not participate any message routing, it can be powered by battery and set to low power mode during the idle time. A typical ZigBee WSN with self-healing function is shown in Figure 2.

The network startup routing route is shown in Figure 2(a). For some reasons, R0, the parent node of E0 and E1, is broken and cannot forward message to $\mathrm{C} 0$ anymore (as shown in Figure 2(b)). When E0 and E1 detect this problem, they rejoin the network via the connection of R1 and R2 accordingly, as shown in Figure 2(c). Connections from $\mathrm{E} 0$ to $\mathrm{C} 0$ and from $\mathrm{E} 1$ to $\mathrm{C} 0$ are repaired automatically. 


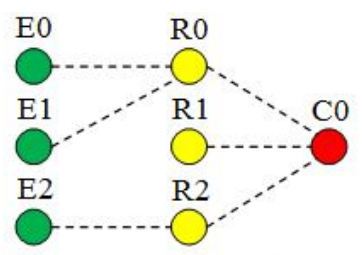

(a) ZigBee wireless sensor network

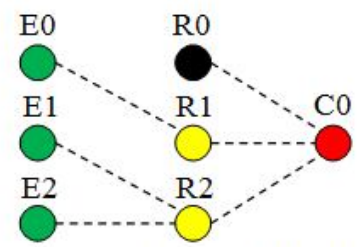

(c) ZigBee network after self-healing

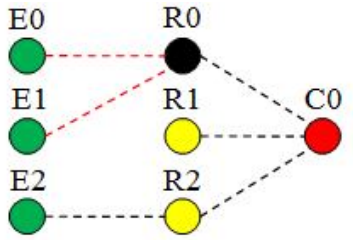

(b) ZigBee network with broken node

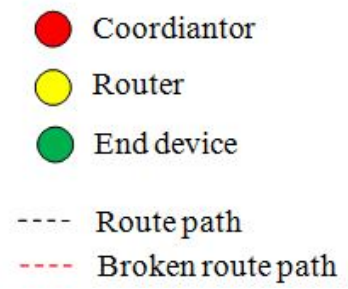

Figure 2. ZigBee network and self-healing function.

\subsection{ZigBee WSN adaptation for heritage preservation purpose}

Basically, the ZigBee protocol can create a large scale, self-organized and self-healing wireless sensor network. Such a network is very suitable for wireless monitoring and control applications. However, some problems emerged when the ZigBee network is practically deployed in cultural buildings. Firstly, a battery-powered end device might lose the network communication for a period. When this problem happens, the ZigBee standard did not define any procedure for the end device to rejoin the network with low power consumption. Secondly, the Ad hoc On-Demand Distance Vector (AODV) routing algorithm utilized by the ZigBee network cannot detect the network communication problem automatically. A ZigBee router can only realize this problem when it is asked to route messages. In other words, the ZigBee standard does not provide any method to guarantee high message transmission reliability. Finally, ZigBee routers are defined as mains-powered. This requirement brings problems to the monitoring system installation for large cultural heritage buildings where power outlets are very limited. The solutions for all mentioned problems are described in Chapter 4. 


\section{General System Description}

The wireless remote monitoring and control system mainly consists of three parts, i.e., the wireless sensor network part, the gateway part and the web service part. Wireless sensor networks are deployed in different cultural buildings. A typical wireless sensor network includes sensor nodes, message relays, control nodes and a data concentrator. A sensor node, named end device in ZigBee standard, reports sensor messages periodically to the data concentrator (named coordinator in ZigBee standard). If a sensor node and the concentrator are out of radio range of each other, one or more message relays, named routers in ZigBee standard, can be deployed in between so that messages can be relayed from the source to the destination. Control nodes are implemented based on router functions. They execute control commands sent from the data concentrator. By connecting radiators or dehumidifiers, control nodes can adjust the indoor climate according to received control commands. The gateway software works for both monitoring and control functions. For monitoring function, the gateway forwards sensor messages from a wireless sensor network to the web service. The gateway also forwards the warning messages which include abnormal sensor readings and wireless device offline status to the web service. For the control function, the gateway receives the climate setting from the web service. It also generates control commands to different control nodes. Different control algorithms are implemented in order to achieve different control results. The web service provides a web-based user interface. The web service collects sensor messages from different locations and represents the sensor data in different curves. Authorized users can login to the webpage remotely to adjust the indoor climate in different cultural buildings. 


\subsection{Remote monitoring system architecture}

The remote monitoring system architecture is shown in Figure 3. Wireless sensor networks are deployed in different buildings. The end device in a WSN reports the temperature and relative humidity information periodically to the network concentrator. The concentrator is connected to a gateway (local manager) via the USB port. Once the local manager receives the sensor message from the wireless sensor network (WSN), it reforms and forwards the message to the web service via the Internet. All local managers communicate with the web service via the Internet. The web service provides graphical presentation of the sensor data via a webpage. After login to the web service, users can remotely monitor the measurement results of different buildings.

WSN Local Manager Data Transfer Web Service Remote User

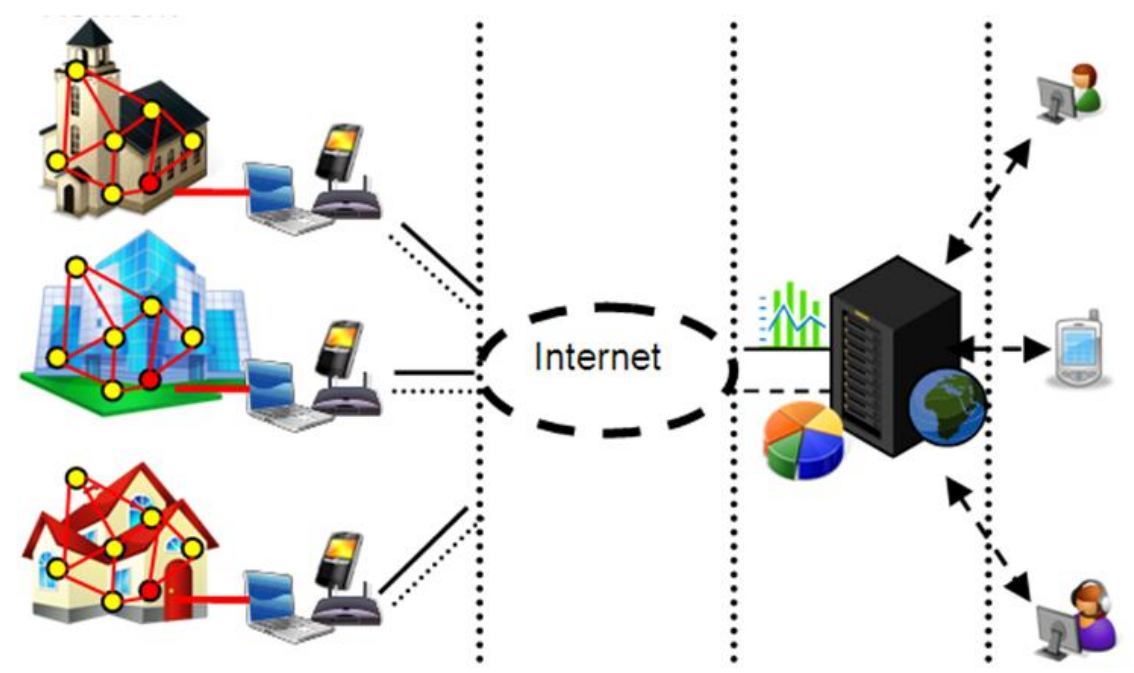

Figure 3. Remote monitoring function description.

\subsection{Remote control function description}

The remote control function maintains the indoor climate of cultural buildings. Figure 4 shows a remote climate control scenario. The wireless control system is deployed in one church building. Router R0 and R1 are programmed as control devices. By connecting with a radiator, R0 controls the indoor temperature. Meanwhile, R1 is connected with a dehumidifier to control the indoor relative humidity. The local 
manager synchronizes the indoor climate setting from the web service all the time. For example, a user logs in to the web service and configures the indoor climate as temperature $24{ }^{\circ} \mathrm{C}$ and relative humidity $50 \%$. The submitted command is stored at the web service until it is fetched by the local manager. When the local manager receives the command, it updates its local climate setting.

WSN

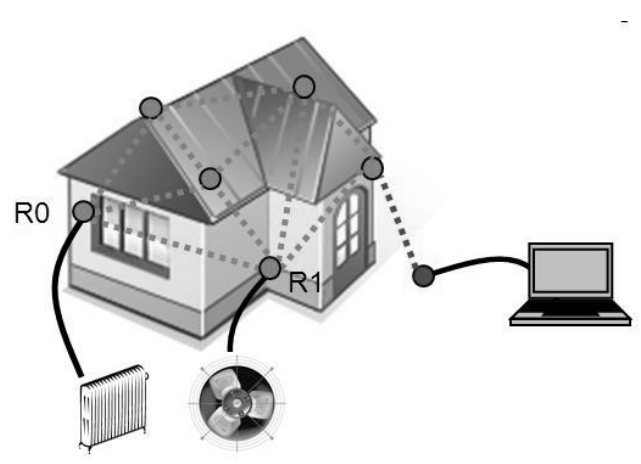

Local Manager

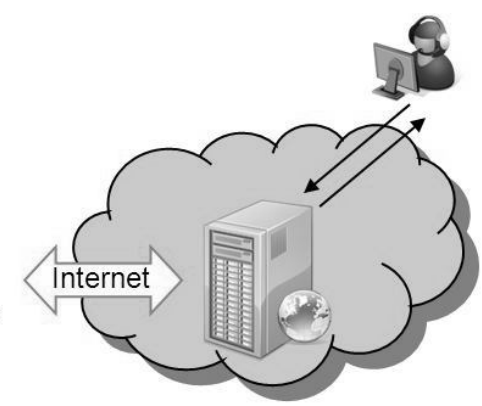

Figure 4. Remote control function description.

However, the wireless control node was not equipped with any sensor which detects the climate change. The sensor messages reported by end devices are taken as the indoor climate reference. Generally, the control command is generated by the local manager based on the climate setting, end device sensor report and control algorithm configuration.

\subsection{Hardware description}

Wireless modules utilized in this study are developed by our research group [58]. The RF chip utilized in the wireless devices is CC2530 and CC2531 from Texas Instrument [59]. In order to increase the radio propagation range, a power amplifier and low noise amplifier chip (PA/LNA) is utilized in all RF modules [60], [61].

Figure 5 shows the outlook and PCB view of the end device module. A temperature and relative humidity sensor, named SHT11 [62], is mounted in the end device. The light emitting diode (LED) in the top right corner indicates the network status and radio 
strength by different blinking combinations during the device deployment. The push button is the hardware reset button of the end device. The end device is powered by a $1 / 2$ AA $3.6 \mathrm{~V}$ Lithium battery with capacity of $1200 \mathrm{mAh}$. A switch in the right side switches ON/OFF the power to the end device.

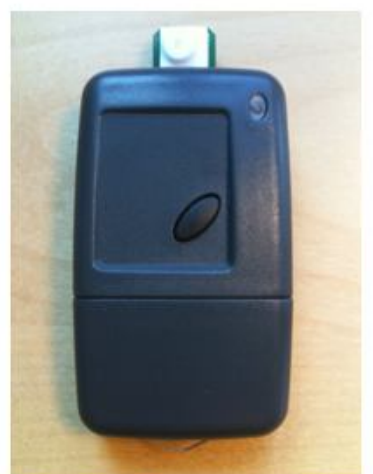

(a) End device

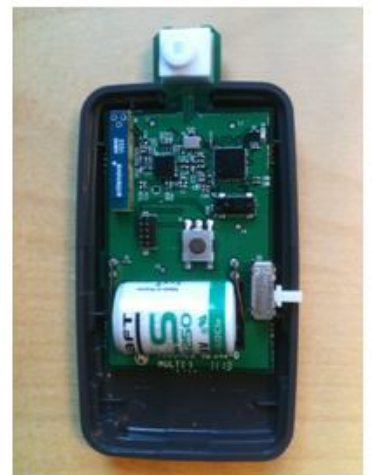

(b) End device PCB

Figure 5. End device of the ZigBee wireless sensor network.

The hardware shown in Figure 6 can be used as a router, a coordinator or the central logic part of the wireless control node. The device is powered from the USB port. When the device is programmed as the coordinator, the USB port is also used to communicate with the local manager. It forwards the message to the local manager and receives the control command from the local manager. If the device is programmed as a router, the USB port is only used as power supply. 


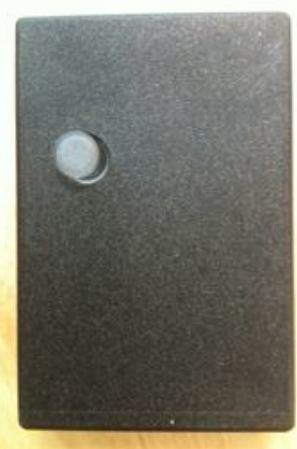

(a) Router

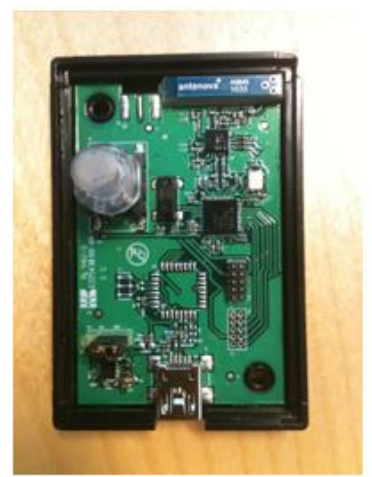

(b) Router PCB

Figure 6. Router of the ZigBee wireless sensor network.

A ZigBee router module can also be programmed as a wireless control node. By connecting with a power relay, the wireless controller can control the power ON/OFF by switching the power relay. Figure 7 shows the wireless controller example. The green LED indicates the network communication status during the control function execution. Additionally, a function switch is implemented to force the controller working at manual $\mathrm{ON}$, manual $\mathrm{OFF}$ or remote control mode. In the manual mode, the wireless controller is forced to be $\mathrm{ON}$ or OFF regardless of the wireless control command. In the remote control mode, the wireless control node is switched according to the wireless control command.

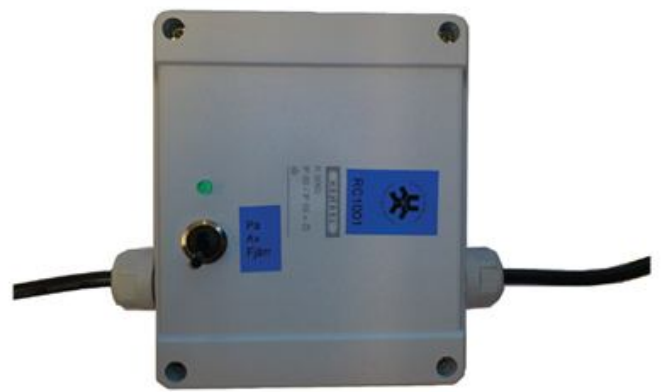

Figure 7. Wireless control node.

Finally, a prototype of battery-powered router is developed for prove of concept. According to the ZigBee standard, a ZigBee router should always be mains-powered. In order to put the ZigBee router into low power mode, a microprocessor, named 
MSP430 [63], is utilized as the Synchronization Controller (SC) which controls the power supply of the ZigBee router according to the WSN applications. The hardware construction of the battery-powered ZigBee router is shown in Figure 8. Internally, MSP430 and ZigBee router communicates via the UART port. The MSP430 controls the power by enable/disable the power regulator of the ZigBee router. If the enable pin of the power regulator is set to "low", the power of the ZigBee router is cut off. Both MSP430 and the ZigBee router are powered by 2 serially connected $1.5 \mathrm{~V}$ AA batteries.

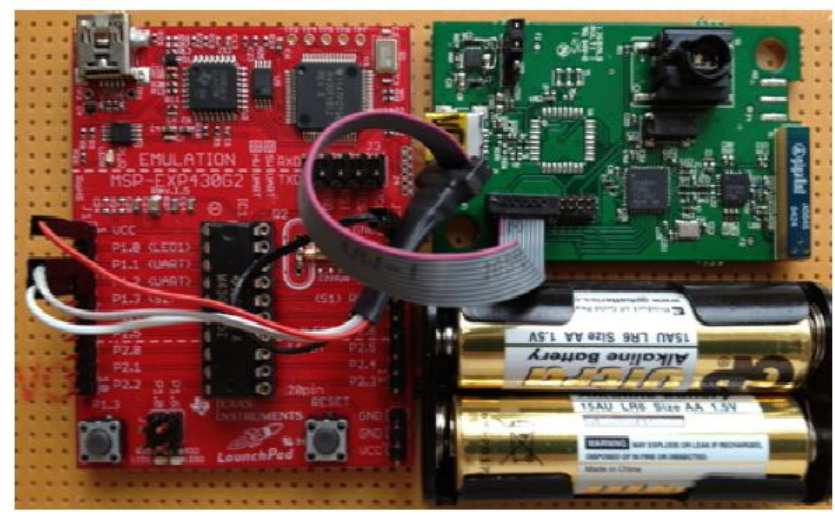

Figure 8. Battery-driven ZigBee router prototype. 


\section{Remote Monitoring and Control System}

This chapter presents the detailed description of the remote wireless monitoring and control system. In the wireless sensor network part, the ZigBee network performance improvement methods are firstly introduced. The monitoring and control functions in the WSN part are developed based on the enhanced wireless sensor network. Additionally, a solution for battery-driven router ZigBee network is presented. In the local manager part, the local manager software architecture is firstly introduced. Monitoring, warning and control function implementations are described in detail. In the web service part, the web service architecture is presented. It also shows the webbased user interface for the remote monitoring and control system.

\subsection{Wireless monitoring and control networks}

The wireless sensor network design and implementation for the remote monitoring and control system is presented in this section. Firstly, the wireless sensor network performance is improved. The message transmission reliability of the WSN is enhanced. End device power consumption is optimized. Secondly, the monitoring and control function implementation is described. Finally, a truly battery-driven wireless sensor network for remote monitoring function is proposed at the end.

\subsubsection{Reliability enhancement}

In the wireless sensor network part, the ZigBee concentrator maintains the communication between the wireless sensor network and the local manager. All end devices send measured sensor data to the concentrator periodically. In order to acquire the message transmission result, an end device can configure the message 
acknowledgement as either end-to-end or peer-to-peer. In the end-to-end method, the acknowledgement is sent from the message destination device. When such acknowledgement is received, the end device knows if the message has been successfully received by the concentrator. In the peer-to-peer method, the end device only receives an acknowledgement sent from its direct parent. The end device does not know if the message has successfully reached the destination device or not. Obviously, from the function point of view, the end-to-end method provides much better reliability. However, this method also introduces overhead into the resource constrained WSN. Firstly, the peer-to-peer method only requires the router routing record from the end device to the destination. However, the end-to-end method requires the routing record in both directions since the end device is supposed to receive the acknowledgement from the concentrator. In such a case, the routing table size of the router is doubled. Secondly, the end-to-end method requires longer waiting time for the acknowledgement. Compared with the peer-to-peer method which only gets the acknowledgement from the direct parent device, the end-to-end method requires the end device to receive a message from the concentrator. The end device waiting time depends on the number of hop from the end device to the concentrator. Such a waiting time can affect the lifetime of the battery-powered end device. Due to these problems, the peer-to-peer acknowledgement method is utilized in most applications. Further message routing tasks are left to the mains-powered routers.

However, the peer-to-peer acknowledgement method has two unsolved problems regarding the message transmission reliability. Firstly, in a multi-hop ZigBee wireless network, the communication problem happened during the route path is invisible to end devices. If the route from a router to the concentrator cannot be repaired by the selfhealing function, the end device sensor messages sent via this router are lost. As shown in Figure 9(a), E0 sends sensor message to C0 periodically. The communication between E0 and R0 is working well. However, the route between R0 and R1 is broken. Since E0 only check the ACK from R0 in the peer to peer acknowledgement, all messages from E0 are blocked by R0. Secondly, a router with broken route might also associate new devices to join the WSN. As shown in Figure 9(b), the broken router R0 might become the message stopper of all devices associated with it. 


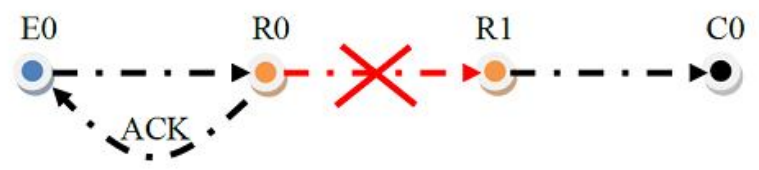

(a) End device message lost due to router communication fail

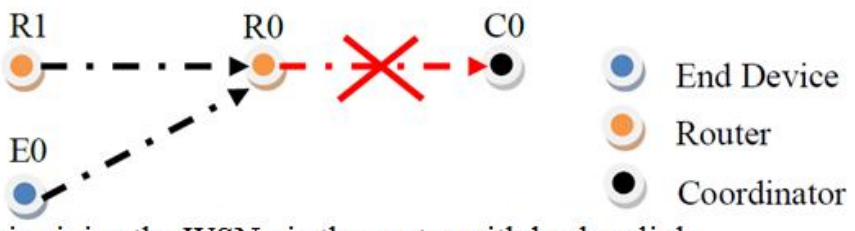

(b) End device joins the WSN via the router with broken link

Figure 9. ZigBee WSN message reliability problems.

To solve these problems, the end device needs to know the communication status of its parent device. Extra heart-beat messages are added for both routers and end devices. As shown in Figure 10, router R0 keeps sending Status_Req request to the concentrator. If the original route via R1 is broken, the self-healing function can help R0 to find new path to the concentrator. Meanwhile, end device E0 keeps sending Status_Req request to its direct parent $\mathrm{R} 0$. If the link between $\mathrm{R} 0$ and $\mathrm{C} 0$ is broken, $\mathrm{E} 0$ receives this notification from R0 directly.

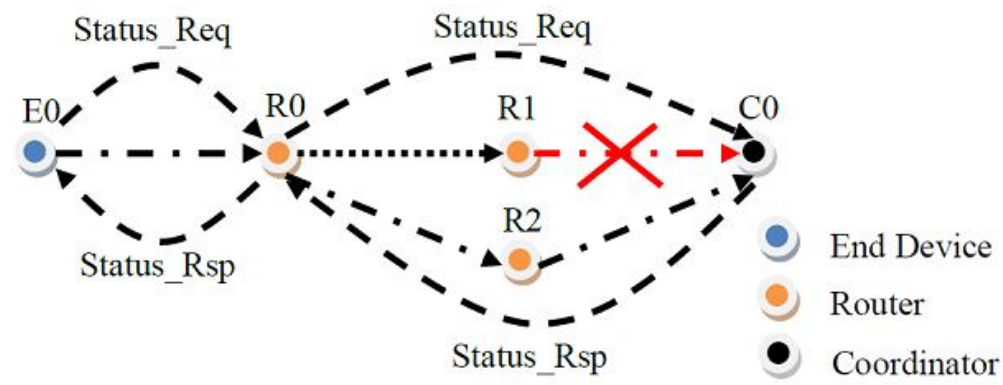

Figure 10. Heart-beat messages added in WSN communication. 
Basically, this design does not save any message communication compared with the end-to-end acknowledgement method. However, this method provides the advantages in the following aspects.

- All routers in the WSN keeps track on their own communication status with the concentrator. If the communication is broken, routers try to recover the communication with the concentrator by using the AODV algorithm all the time.

- The communication status check is initiated by routers that are mains-powered.

- This method provides an interface for other device to query the communication state of the router.

- By querying the communication status from the direct parent device, the end device increases the possibility of successfully sending sensor message to the concentrator.

The possible communication problems from an end device to the concentrator are described in Figure 11. The end device E0 communicates with the concentrator $\mathrm{C} 0$ via router R0. In Figure 11(a), the communication between E0 and the parent device R0 is broken. Such a problem can be directly detected by the peer-to-peer acknowledgement. In Figure 11(b), E0 can communicate with the parent device R0. However, the communication between $\mathrm{R} 0$ and the concentrator $\mathrm{C} 0$ is broken. As described in Figure 10 , such a problem can be detected by the heart-beat message sent from R0. When end device E0 sends Status_Req request to R0, this communication problem is acknowledged from R0 to E0 in the Status_Rsp.

Practically, the communication might take very long time to recover. The end device is designed to actively find alternative parent devices if it keeps detecting the communication problem to the concentrator via the current parent device. As shown in Figure 11, the NWK_Disc message is sent by the end device. The NWK_Disc is a broadcast message which detects all routers or the concentrator within the end device radio propagation range. The router or the concentrator responds the NWK_Disc with Disc_Rsp message. The Disc_Rsp message contains a flag which indicates the communication status from the device to the concentrator. The end device analyzes the received Disc_Rsp messages. If a router with working communication route to the concentrator is found, the end device rejoins the WSN via the association with that router. As shown in Figure 11(a), E0 only receives the Disc_Rsp from R1. It is because 
that the communication between E0 and R0 is broken. Moreover, R1 can communicate with $\mathrm{C} 0$ via R0. As a result, E0 rejoins the WSN via the association to R1. As shown in Figure 11(b), the Disc_Rsp is received by E0 from both R0 and R1. However, R0 does not have a communication route to $\mathrm{C} 0$. As a result, the Disc_Rsp sent from R0 indicates the communication problem between $\mathrm{R} 0$ and $\mathrm{C} 0$. When E0 receives the Disc_Rsp from R0, the router R0 is not considered as the alternative parent candidate. However, R1 can communicate directly with $\mathrm{C} 0$. The end device rejoins the WSN via the association to R1 instead.

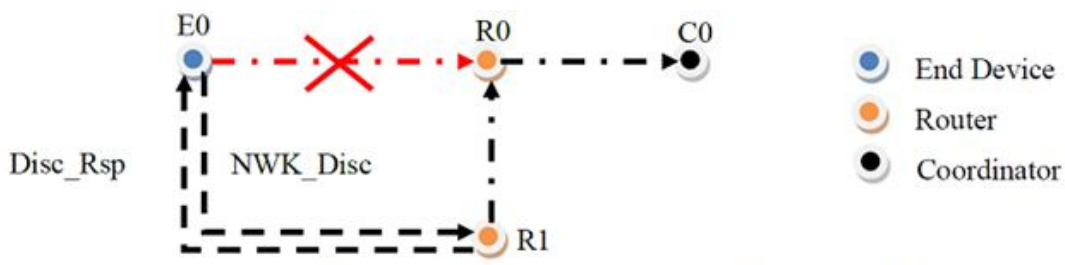

(a) End device lost connection with direct parent

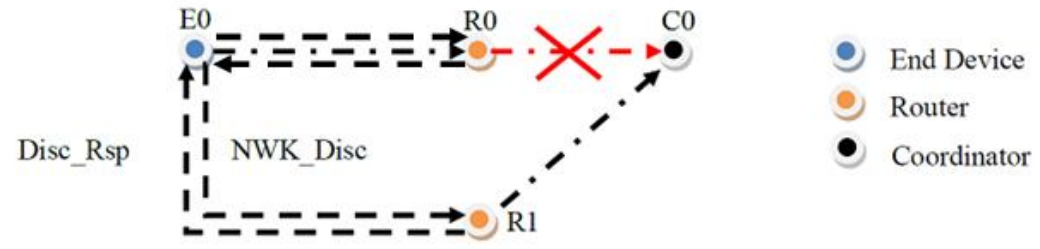

(b) Router communication problem lead end device to find new "parent"

Figure 11. End device discovers the network before rejoin the network. 


\subsubsection{Router firmware design}

The router firmware implements the message reliability enhancement method described in 4.1.1. Enhancement operations are controlled by a state machine as shown in Figure 12.

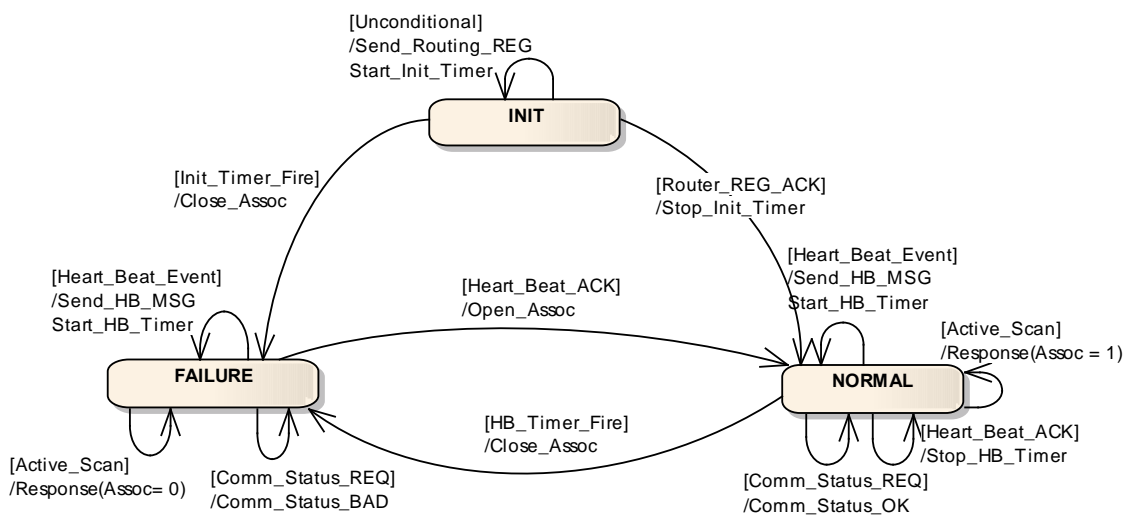

Figure 12. Router reliability enhancement state machine.

The router works at the INIT state when it joins the WSN. In the INIT state, the router sends out the Router Registration message to the concentrator. This message aims to create a message route from the router to the concentrator. Meanwhile, a timer called Start_Init_Timer is created to define the maximal waiting time of the registration acknowledgement. When the router receives the Registration Acknowledgment from the concentrator, the state machine jumps to the NORMAL state. If the acknowledgement is not received by the router before the Start_Init_Timer fired, the state machine jumps to the FAILURE state. Meanwhile, the MAC_ASSOC is switched off so that the router cannot associate any new device into the network. In the NORMAL state, the router sends Heart_Beat_MSG to the concentrator periodically. If the Heart Beat ACK is received by the router, the state machine stays at the NORMAL state. Otherwise, the state machine jumps to the FAILURE state. When the router works at the NORMAL state, it responses the Communication Status Query sent from end devices. Comm_Status_OK indicates that the router maintains a working communication link to the concentrator. In the FAILURE state, the router always tries to repair the connection by sending "Heart Beat Message" to the concentrator. Once the 
router receives the Heart_Beat_ACK from the concentrator, the state machine jumps back to the NORMAL state. The "MAC_ASSOC" is reopened. If the router receives a “Communication Status Query" in the FAILURE state, the router responses the end device with Comm_Status_BAD. It is a message which indicates that the link from the router to the coordinator is broken.

The heart-beat message interval is configurable in this state machine. If the system needs to know the communication status of the router all the time, a higher heart-beat sending rate can be configured. Otherwise, the heart-beat message can be sent just before the end device sending the sensor message to the concentrator. In such a case, the heart-beat message works more like a hand-shake message.

\subsubsection{End device firmware design}

The end device firmware consists of two parts, the end device sensor working state machine and the end device high availability state machine. As a battery-driven device, the power consumption is also considered in the state machine implementations. In the end device senor working state machine, the measured sensor data is compared with the historical data, if two measured data are within the message sending threshold, the end device goes to "sleep mode" without sending the sensor message to the concentrator. The end device high availability state machine keeps detecting the communication status of the parent device. If the parent device cannot maintains a route with the concentrator, the end device looks for other routers within the radio propagation range. If another router with working route to the concentrator is found, the end device rejoins the network by association of that router.

\subsubsection{End device sensor working state machine}

Figure 13 shows the end device sensor working state machine. The state machine works at the NWK_ESTABLISHED state when the end device joins the network. If the SENSOR_WORK_EVENT is triggered, the end device collects the temperature and relative humidity data and jumps to the TEMP_HUMI_SENSED state. The newly collected sensor value is compared with the old value stored from the last measurement. If the new value is close to the old value (e.g., if the deviation is within $\pm 0.3{ }^{\circ} \mathrm{C}$ range) 
the end device does not send out the sensor message but goes to the POWER_SAVING state directly. Otherwise a new message is formed and the state machine goes to the MSG_PKT_FORMED state. In this state, the end device sends the sensor data message to the concentrator. If the message is sent successfully, the state machine goes to the PKG_SENT state and automatically jumps to the POWER_SAVING state. In the POWER_SAVING state, the end device configures the timer for the next sensor reading and goes to sleep mode.

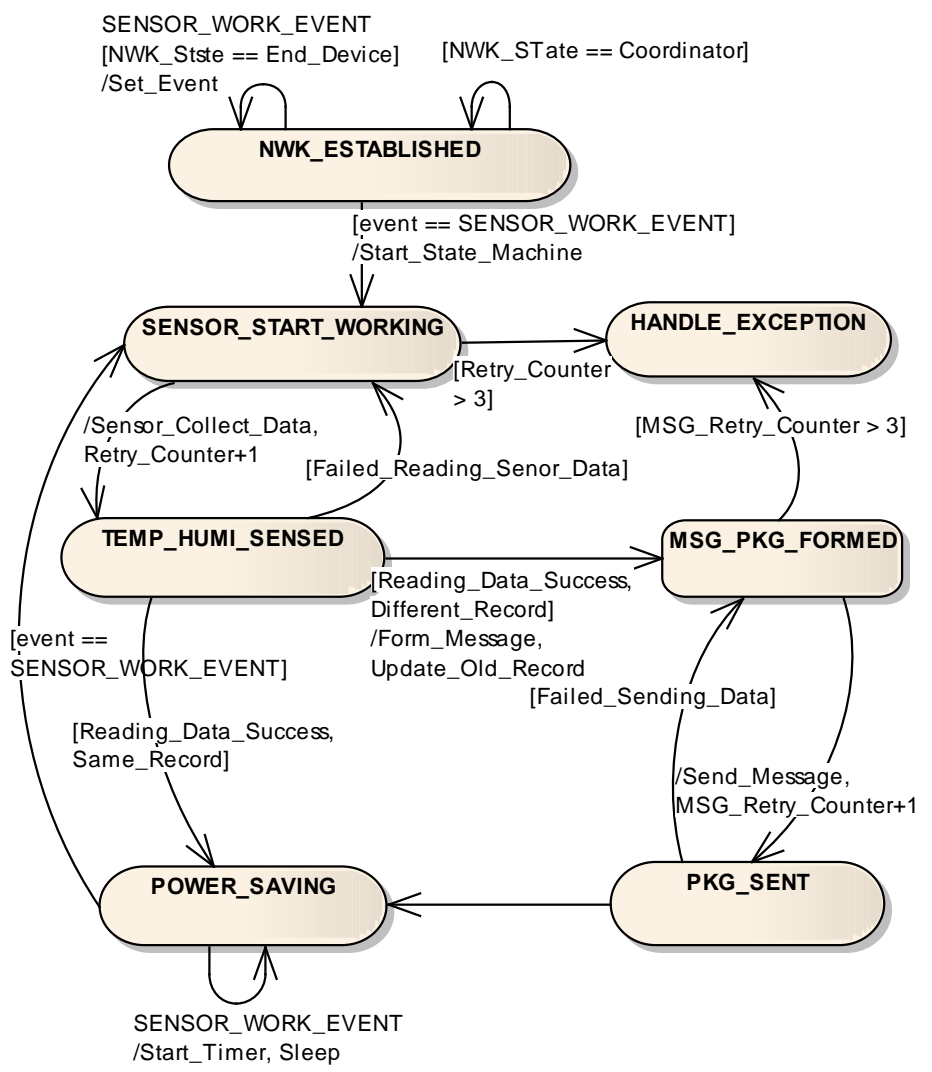

Figure 13. End device sensor working state machine.

When storing the historical value at the TEMP_HUMI_SENSED state, it is important to assign the old value only at the MSG_PKT_FORMED state. This is to prevent the miss-reporting of the sensor data. For example, the temperature reporting threshold is set to $0.3{ }^{\circ} \mathrm{C}$. The end device sensor detects 3 consecutive temperature changes: 
$11.5{ }^{\circ} \mathrm{C}, 11.7{ }^{\circ} \mathrm{C}$ and $11.9{ }^{\circ} \mathrm{C}$. If the old value is updated each time when the temperature is measured, $11.9^{\circ} \mathrm{C}$ will not be reported to the concentrator since $11.9{ }^{\circ} \mathrm{C}$ is checked against $11.7{ }^{\circ} \mathrm{C}$. The $0.2{ }^{\circ} \mathrm{C}$ deviation is inside the threshold. As a result, the sensor loses the sensitivity of detecting the smooth temperature change. However, if the old value is only assigned when it jumps to the MSG_PKG _FORMED state, $11.9^{\circ} \mathrm{C}$ will be reported since the historical value is $11.5^{\circ} \mathrm{C}$.

Furthermore, sensor module exceptions are also handled in the state machine, as showed in Figure 13. The state machine goes to the HANDLE_EXCEPTION state when the sensor fails to collect the sensor information for three times or the sensor module fails to send out data after retrying 3 times.

\subsubsection{End device high reliability state machine}

The end device high reliability state machine is developed to enhance the message transmission reliability for the end device with low power consumption. As shown in Figure 14, four states are defined in the state machine, which are INIT, NORMAL, SYNC and FAILURE state. The end device works in different states according to the end device network communication status. Generally, the state machine provides the following functions.

- Keep detecting the communication status of the parent device.

- Buffer measured sensor messages when end device lost the communication with the concentrator.

- Upload buffered sensor messages to the concentrator when the route to the concentrator is reestablished.

- Rejoin the network if the parent device cannot repair the route with the concentrator.

- Go to low power mode during the idle time and save power. 


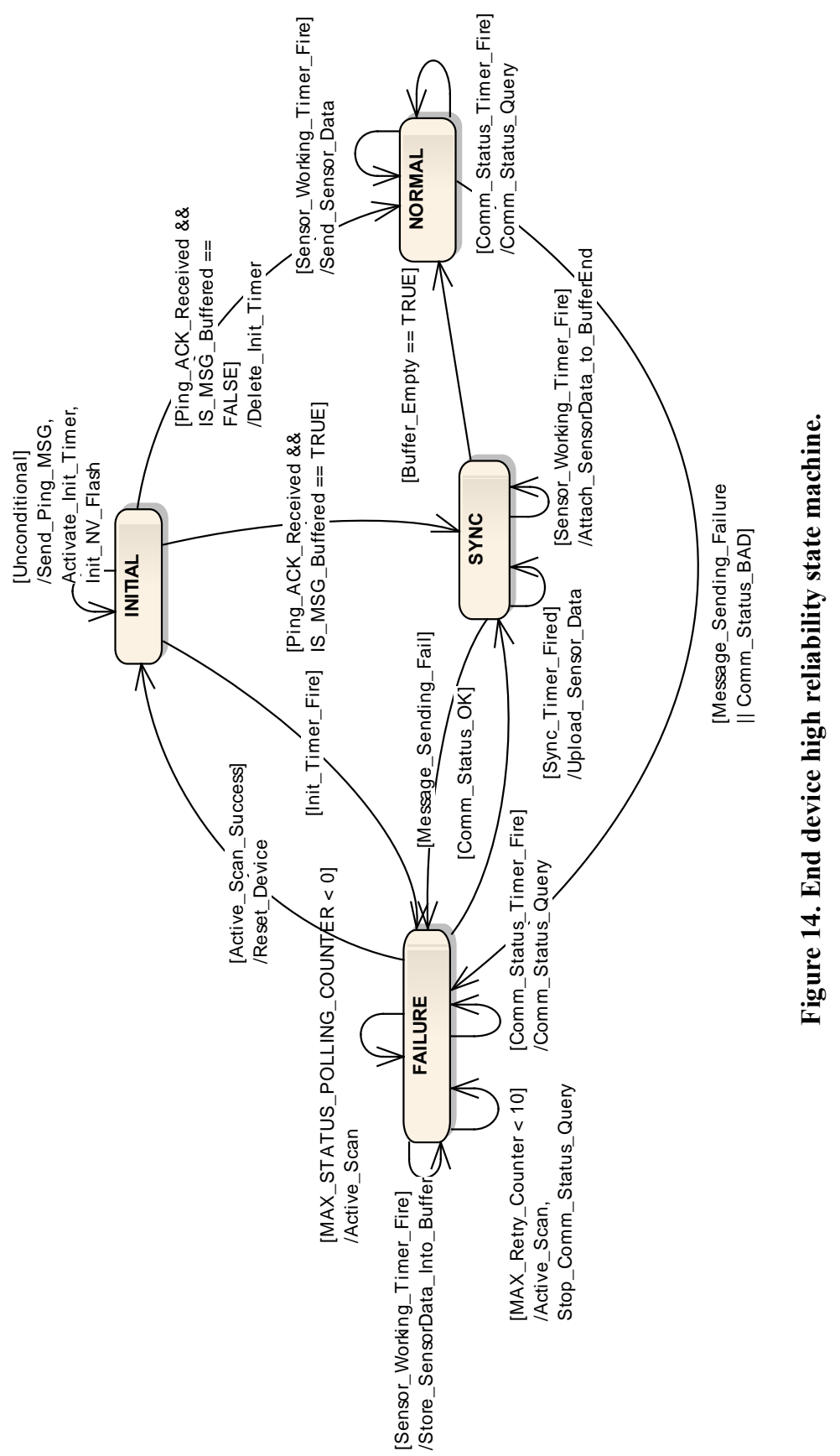




\section{INITIAL State:}

The state machine works at the INITIAL state after the end device joins the wireless network. In this state, the end device firstly sends a PING message to the concentrator. At the same time, a timer is started with the "timeout" interval which defines the maximum waiting time for the PING message. Once the concentrator receives this PING message, it responses to the end device with an acknowledgement. If the acknowledgement is not received before the Init timer fired, the state machine jumps to the FAILURE state. Otherwise, the end device initializes its flash memory to check if there is any buffered sensor message. If the flash memory is empty, the state machine jumps to the NORMAL state. Otherwise, it jumps to the SYNC state to upload buffered messages. The PING message is only sent when the end device joins the network. It helps the end device to establish a route to the concentrator. The routers in the network are configured to never age the route record [64]. In such a case, the communication link from the end device to the coordinator is always established before any sensor message is sent.

\section{NORMAL State:}

When the end device works at the NORMAL state, it periodically collects the sensor reading, repacks the sensor message and sends the sensor message to the concentrator. The acknowledgement of the sensor information (Peer-to-Peer) is checked after each sensor information transmission. If the end device fails to send the message to its direct parent, the state machine jumps to the FAILURE state. The end device also sends Comm_Status_Query to its direct parent periodically. When receiving this query, the parent of the end device responses with its communication status to the concentrator. If the parent replies the end device with COMM_STATUS_BAD, the end device state machine jumps to the FAILURE state. Otherwise, the state machine stays at the NORMAL state.

\section{SYNC State:}

SYNC is the abbreviation of synchronization. In the SYNC state, the buffered sensor messages are uploaded from an end device to the concentrator. The end device keeps uploading sensor messages until the buffer is empty. When the buffer is empty, the end device state machine jumps to the NORMAL state. During the message uploading, the end device also collects the sensor information. The newly collected sensor information 
is appended to the end of the message buffer to keep the correct message sequence. Moreover, the message transmission result is checked for each uploaded message. If the message fails to transmit from the end device to its direct parent, the state machine jumps to the FAILURE state.

\section{FAILURE State:}

All end device exceptions are handled in the FAILURE state. If the end device detects any communication problem, it jumps to the FAILURE state. The end device keeps collecting sensor information in the FAILURE state, the collected sensor information is saved in the sensor message buffer of the end device. Meanwhile, the end device sends Comm_Status_Req periodically. If the received Comm_Status_Rsp indicates the link is recovered, the end device state machine jumps to the SYNC state and synchronizes the buffered messages. A counter is also configured in the FIALURE state. The counter decreases by 1 each time if the end device fails to communicate with its direct parent. When the counter reaches 0 , the end device considers that the current link is unrecoverable. Then the end device tries to find a new parent by sending the discovery command over the network periodically. In this procedure, a standard ZigBee command called Network Discovery is sent from the end device as a broadcast message. All routers within the radio propagation range of the end device response this command. When the end device receives the command response, the MAC_ASSOC identifier in the response of each router is checked. If the response includes a router with open MAC_ASSOC, the end device resets itself and rejoins the WSN via the association of that router.

The end device sensor working state machine and end device high reliability state machine runs in parallel in the end device firmware. Both state machines are triggered by different events and can be executed independently. However, the execution result of one state machine can be the trigger for each other. For example, if an end device fails to send sensor message to the parent device, both state machines are triggered.

Additionally, these two state machines also define the general behavior of batterypowered wireless sensor node. They are designed and implemented independent of any sensor types. Different sensor drivers can be loaded by these two state machines and provide same message transmission reliabilities. 


\subsubsection{WSN control function Implementation}

The control function is developed based on the ZigBee WSN with enhanced reliability. Since the major logic of the control function is implemented in the local manager, the control function in the WSN part is developed to only obey the control command sent from the local manager. Figure 15 shows the control function message flow. In order to increase the communication reliability, all devices in the wireless sensor network run together with the stability enhancement state machine.

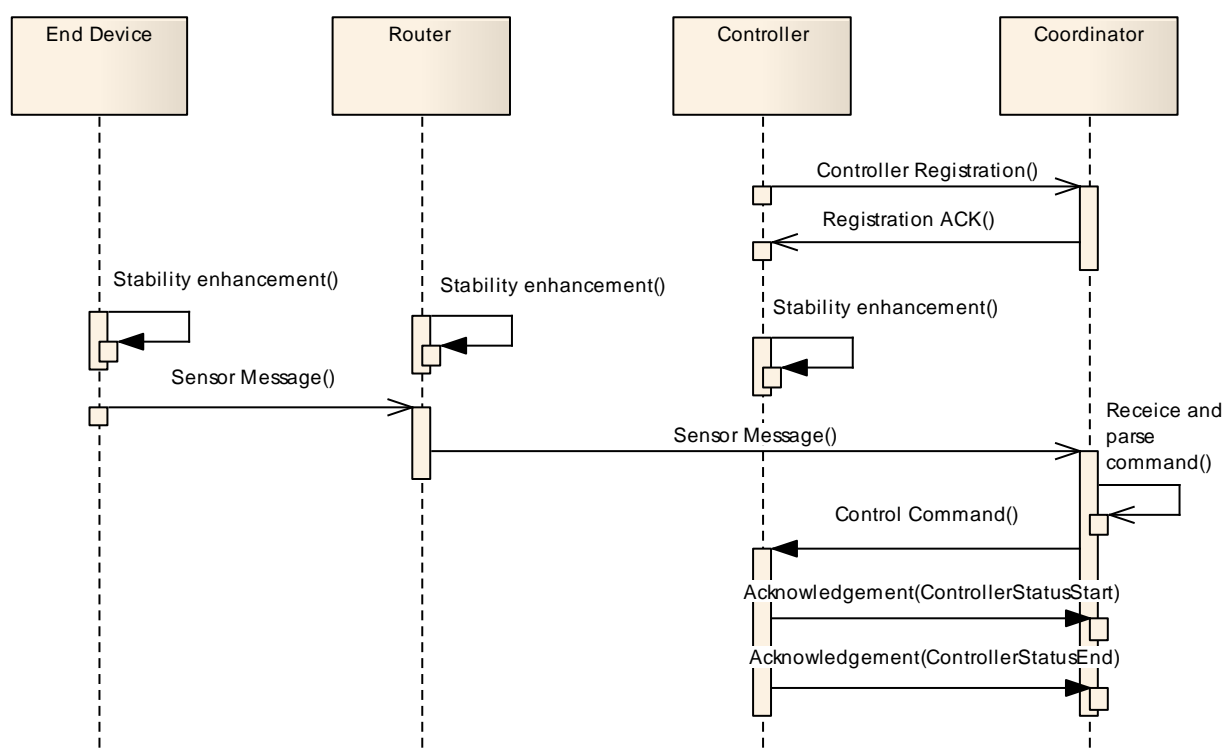

Figure 15. Control command generation and execution.

The controller registration message is sent by the wireless control node which newly joins the wireless sensor network. This message provides the wireless controller description to the local manager. When the local manager receives this message, it saves the registration information into its database. The local wireless control process is always triggered by the feedback sensor message. Feedback end devices are programmed to periodically send temperature and relative humidity messages to the local manager. After calculation, the local manager sends back the control command to the concentrator as described in Figure 16. The Message Header field identifies the command type. The "Network Address" field contains the address of the wireless 
control node. The Interval field contains the interval (in seconds) that the wireless controller should switch on the power relay. Once the concentrator receives the command, it forwards the message to the wireless control node whose network address is specified in the network address field. When the wireless controller receives the command, it turns on the power switch with the interval specified in the command. The wireless control node sends two ACKs to the local manager for one control command. The first ACK is sent directly after the control command is received by the wireless control node. The second ACK is send when the turn on interval specified in the control command is expired. The controller ON/OFF status is carried inside the ACK message. If the wireless control node stops working, the error code is also carried in the ACK message and forwarded to the local manager. In this way, the wireless control node execution is always under surveillance by the local manager.

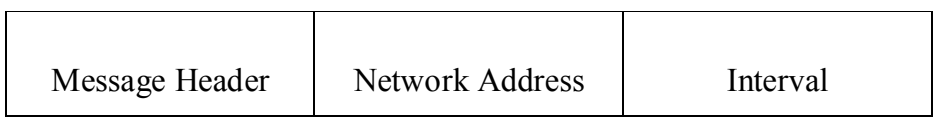

Figure 16. Control command message frame

Control function reliability is highly emphasized in the wireless control networks. All control nodes check the communication status with the concentrator all the time. If a controller loses the communication, no more control operation will be done before the connection is reestablished. This is due to the fact that the cultural heritages, e.g., paintings or wood furniture are more sensitive to the big climate changes [65]. A controller might cause un-recoverable damage without remote access and monitoring. As shown in Figure 16, instead of an ON/OFF command, the turn on interval is sent to the wireless control node. This method eliminates the potential problem when communication between the wireless control node and the concentrator cannot be repaired. For example, if the system utilizes the ON/OFF command and one radiator is turned on by the wireless control node. If the wireless control node loses the communication with the concentrator, the radiator cannot be turned off remotely.

Additionally, a centralized control method is implemented in the system as shown in Figure 17(a). Sensor messages are received by the local manager. Control commands are generated by the local manager and sent to control nodes. Alternatively, as shown 
in Figure 17(b), the wireless sensor network could use end device bind [66] method so that end device could send sensor message directly to the control node. Compared with the centralized control solution described in Figure 17(a), the control with binding end device solution can work independent of the local manager. If the control node loses the communication with the concentrator, the controller can still work according to the current setting by receiving the sensor feedback from the bound end device. However, this advantage could also become a reliability problem. If the controller lost the communication with the local manager, it also lost the possible control command update. Moreover, in order to implement the control method as described in Figure 17(b), each control node should maintain control commands and scheduled tasks. It is better to implement such a heavy calculation in the local manager which is much more powerful than embedded devices.

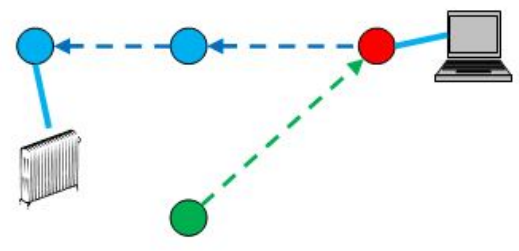

(a) Centralized Control

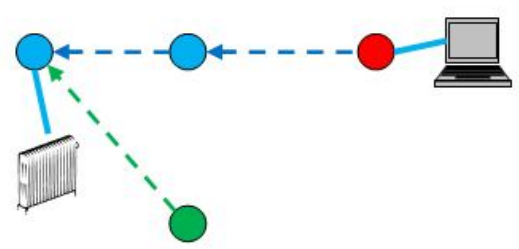

(b) Control with "bind" end device

Figure 17. Different sensor message feedback methods.

Moreover, the sensor message feedback rate for the control function is every 3 minutes. Compared with other industry closed-loop control system, the feedback rate is very low. However, an ordinary heating system usually has a much longer response time than 3 minutes. A large indoor environment, like churches, could take several hours heating to increase $1{ }^{\circ} \mathrm{C}$. In this case, the 3 -minute interval is acceptable for most buildings. Otherwise, users could also configure the sensor report interval according to their specific requirements.

\subsubsection{Truly battery-driven ZigBee network}

In ZigBee standard, The ZigBee router is defined to be mains-powered in order to maintain mesh network features. However, in most cultural buildings, there are not enough power outlets for ZigBee routers. As a result, the network scale and the 
reliability are largely affected. On the other hand, the indoor climate monitoring for the cultural buildings has its own characteristics. Firstly, the measured object does not move all the time. Secondly, the data transmission rate is relatively low. This implies that a ZigBee network utilized for indoor climate monitoring does not have high requirement on the mesh feature provided by the ZigBee network. If the message transmission is scheduled, the router can be put into sleep mode during the idle time. A battery-powered ZigBee router can be implemented.

An extra microprocessor, MSP430, is utilized as the synchronization controller of the ZigBee router. It communicates with the ZigBee router via the UART port. The MSP430 controls the power of the ZigBee router by enable/disable the router power regulator. During the time when the power of the ZigBee router is switched off, the WSN applications of the ZigBee router are maintained by the MSP430 program. It powers on the ZigBee router at different time for different WSN applications. Moreover, the router needs to buffer sensor messages received from end devices. Practically, the flash memory of the ZigBee router can only be written around 2000 times. In this case, the MSP430 needs to provide a sensor message buffer in RAM.

The software program of MSP430 is designed as shown in Figure 18. The Serial Port Interface module handles the UART communication with the ZigBee router. The Message Worker module works as a message decoder. When a UART message is received, the Message worker forwards the message to the correct module according to the message type. The Software Timer module maintains the time intervals of WSN applications for the ZigBee router. One specific application runs as a stand-alone timer item inside the Software Timer. Timer items can be added or removed by the router via the UART communication. When a timer item fires, the power of the ZigBee router is turned on by the Power Switch module. The Sensor Message Buffer module provides the buffer function that saves sensor messages received by the router. 


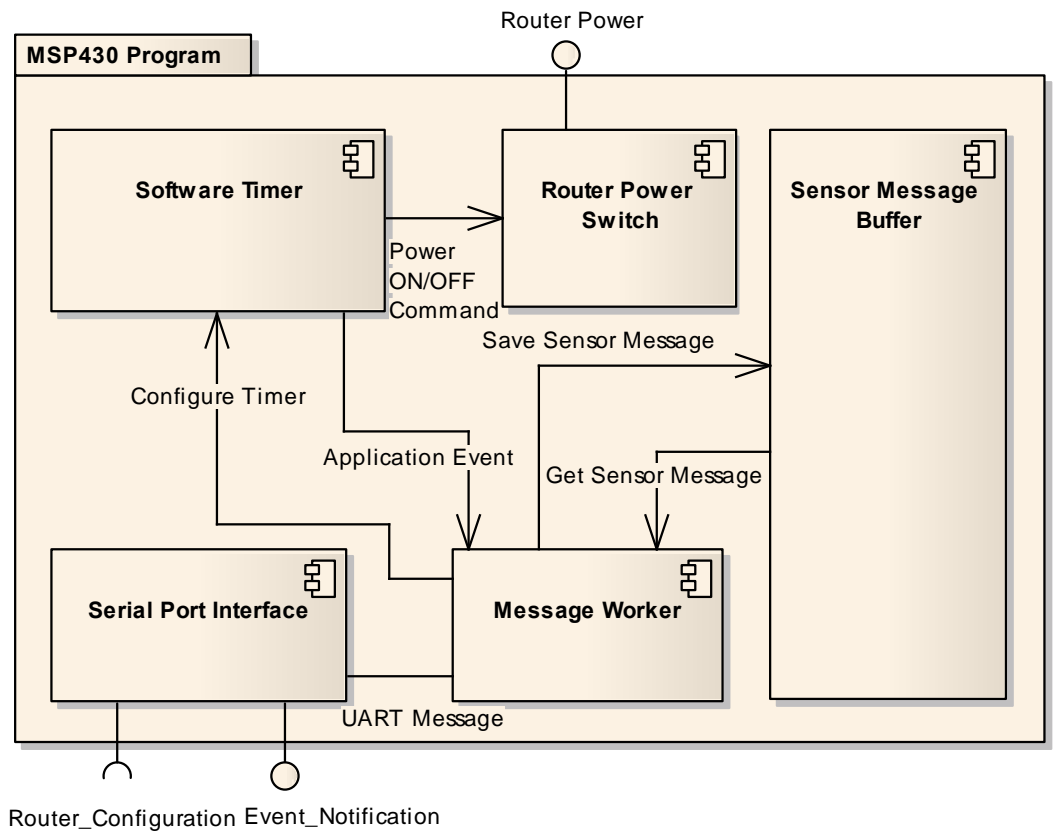

Figure 18. Synchronization controller software architecture.

In order to maximize the router battery lifetime, the router needs to be in low power mode as long as possible. This implies that all application messages should be sent within a short period. In order to overcome possible message collisions, sensor message reporting from different end devices are scheduled in different time slots. Generally, sensor message uploading from the end device to the concentrator is divided into two stages. In the first stage, end devices report sensor messages to the direct parent router. Before going sleep, end devices receive the next sensor report timestamp from the router. In the second stage, all routers in the WSN wake up at the same time. The concentrator sends message query to each router to collect all buffered sensor messages. The sequence of the message fetching depends on the network route. When the sensor message uploading is finished, the router goes to low power mode.

Figure 19 describes the end device time slot allocation mechanism. Extra communications are designed for the router to collect the end device network information. The collected information, i.e., network address and the end device ID are saved in the End Device Address Book component in the router. The router is waked 
up by MSP430 before the first end device sending sensor message. As long as the router receives the sensor message, a command which contains the time stamps for the next sensor message reporting is sent to the end device. In the command, an offset value is assigned which indicates the time slots number. For each end device, a $150 \mathrm{~ms}$ interval is reserved for the sensor message reporting. In this way, the router arranges the sensor message reporting so that no collision could happen.

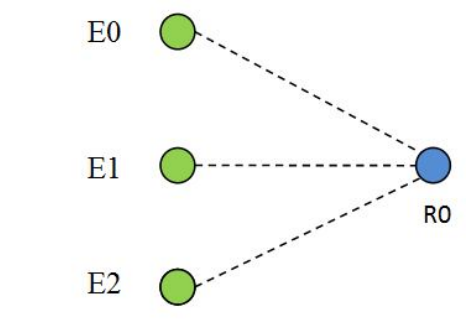

End Device
Router

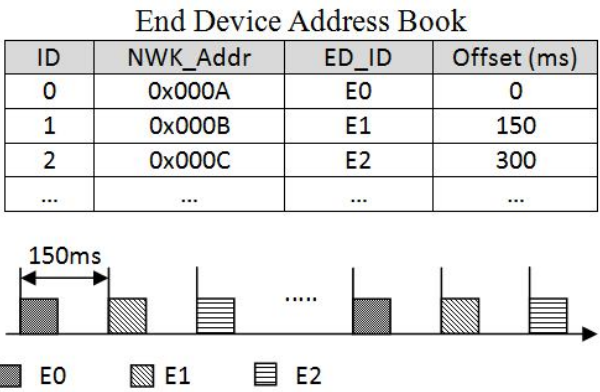

$\square$ EO $\mathbb{Q} E 1 \quad$ 目 E2

Figure 19. End device router communication scheme.

In order to keep the mesh feature of the ZigBee network, all routers wake up at the same time when uploading buffered sensor messages to the concentrator. Figure 20 shows the message uploading process. When all routers wake up, the concentrator sends message fetching command to each router. If a router receives this command, it uploads all buffered sensor messages to the concentrator. In order to save the battery power, the router goes to the low power mode after uploading all buffered sensor messages. In this case, the concentrator must fetch a router that is not utilized by other router for message routing. In order to acquire route record from different routers, the concentrator sends a Many-to-One (MTO) routing request to all routers. This message is utilized to create the route path to the concentrator in all routers. When a router receives this routing request, it immediately sends a dummy message to the concentrator. Together with the dummy message, the route path is recorded from the source router to the concentrator. The path information is saved in a message called Rout Record Command (RREC). Figure 20(b), show one example of the RREC creation from $\mathrm{R} 0$ to the concentrator. When the dummy message first sent from R0, the RREC command is empty. After the first message forwarded by R3 (first hop), the network address of R3 is added into the RREC. When the message reaches R5 (second 
hop), the network address of R5 is added into the RREC. The RREC message from each router is saved in the concentrator in the Concentrator Route Relay Table as shown in Figure 20(c). The algorithm that calculates the router message fetching sequence is developed as shown in Figure 20(d).

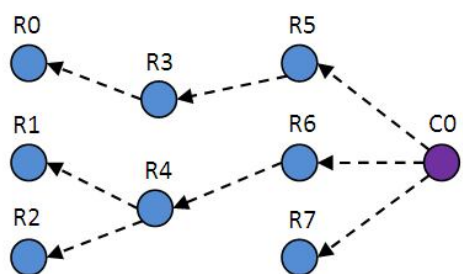

a. Concentrator sends MTO Reqeust

\begin{tabular}{|l|l|}
\hline Src Node & Route Relay List \\
\hline R0 & R3,R5 \\
\hline R1 & R4,R6 \\
\hline R2 & R4,R6 \\
\hline R3 & R5 \\
\hline R4 & R6 \\
\hline R5 & 0 \\
\hline R6 & 0 \\
\hline R7 & 0 \\
\hline
\end{tabular}

c. Concentrator route relay table

\begin{tabular}{|l|l|l|l|l|l|l|l|}
\hline $1^{\text {st }}$ & $2^{\text {nd }}$ & $3^{\text {rd }}$ & $4^{\text {th }}$ & $5^{\text {th }}$ & $6^{\text {th }}$ & $7^{\text {th }}$ & $8^{\text {th }}$ \\
\hline R0 & R1 & R2 & R7 & R3 & R4 & R5 & R6 \\
\hline
\end{tabular}

e. Router message fetching sequence Array

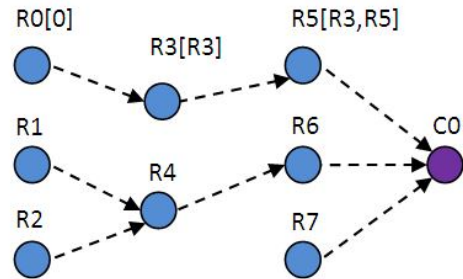

b. Router RREC creation

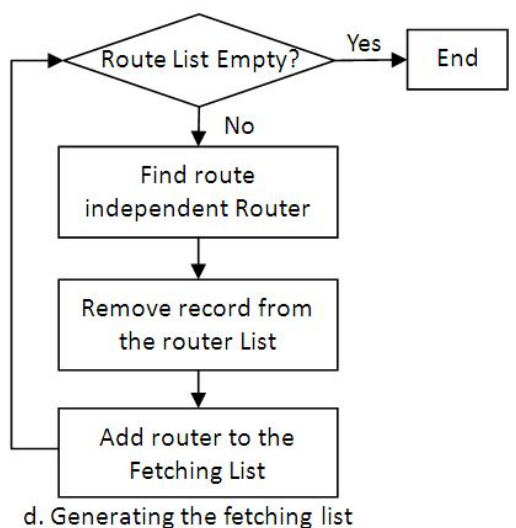

Figure 20. Router - concentrator communication scheme.

The algorithm keeps going through the Concentrator Route Relay Table. The table is scanned row by row in order to find one router whose network address is not included in the Route Relay List of any other routers (find the route independent router). Once the router is found, the corresponding record is removed from the Concentrator Route Relay Table. The selected router address is appended in the Router message fetching sequence array (shown in Figure 20(e)). The program keeps going through the table until the table is empty. This procedure is done every time before the concentrator starts the sensor message fetching from routers. Although this method introduces a 
little overhead, it guarantees the concentrator to utilize the up-to-date routing information to fetch the message from different routers every time.

\subsection{Local manager}

In the remote monitoring and control system, a local manager mainly plays three roles, the message bridge between wireless sensor networks and the web service, the warning message generator and the logic center of the wireless control network. As a message bridge, the local manager communicates with the WSN concentrator via the USB port, as shown in Figure 21. Meanwhile, the local manager also communicates with the web service via the Internet. In the warning function, sensor messages received by the local manager are checked against predefined sensor value thresholds. If a sensor message value is outside the configured threshold, the local manager generates a sensor warning message. The local manager also keeps track of the communication status of all wireless devices in the WSN. If no message update received from a wireless device for long time, a device offline warning is generated by the local manager. In the control function, the local manager works as the brain for the indoor climate control in a cultural building. The local manager synchronizes the building climate setting with the web service all the time. Different control algorithms are implemented in the local manager as well. For a local wireless control system, the local manager maintains all controller related configurations. The control commands are sent to different wireless controllers periodically based on the feedback sensor readings. Additionally, the wireless control function is implemented with control reliability enhancement. 


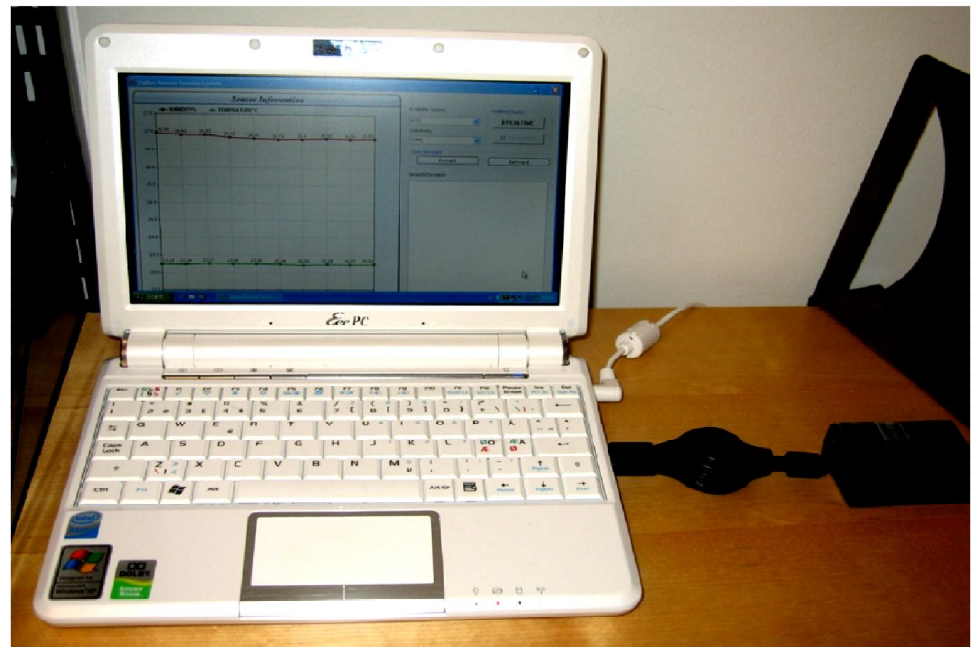

Figure 21. Local manager communicate with concentrator via USB port.

The local manager software is implemented as a Windows program based on Microsoft Foundation Class (MFC) [67]. This solution is developed aiming at fast prototyping and prove of concept. More than often, the Windows operating system has been criticized by stability problems when utilized in long term maintenance-free applications. In this case, an embedded PC running a Linux operating system could be a better choice. Compared with Windows system, the Linux system requires much lower hardware profile. Enormous open source projects based on Linux provide a huge resource library on function development. Moreover, the Linux system can be explicitly tailored according to the user requirement. Nevertheless, one advantage of the Windows-based local manager is that the local manager software can be installed in the existing computers of the cultural buildings since the Windows operating system is still the major operating system utilized.

\subsubsection{Local manager software architecture}

The software architecture is shown in Figure 22. The Serial Port Manager handles serial port communication between the concentrator and the local manager. The DataCommand Synchronizer maintains a two-way data channel between the local manager and the web service. It helps the local manager forwarding the sensor message to the web service. It also helps the local manager to udpate climate setting from the web 
service. The Database Worker component provides database service via ODBC interfaces [67]. Instead of configuration files which are commonly utilized for message recording, a MySQL [68] database is installed in the local manager. This is due to the fact that the local manager is designed to maintain large data and relatively complicated data relationships. A detailed description of the local manager control function database design is presented in section 4.2.3. The Message Broker layer manages all messages related issues in the local manager. The String Parser component forwards the message to the correct component according to the message header. The Device Status List component records the device communication status. The Message Buffer component buffers all messages to the web service if the Internet of the local manager is down. Different application functions are developed based on this infrastructure. At the moment, the monitoring function, warning function and control function are implemented in the local manager.

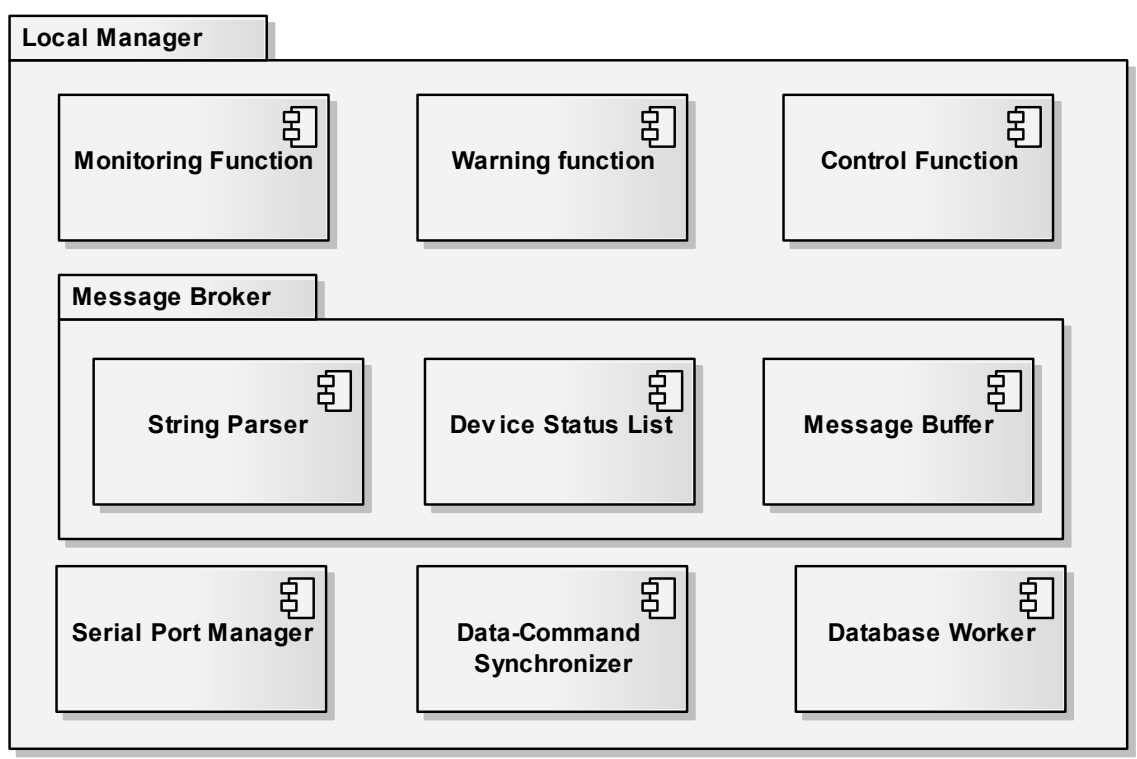

Figure 22. Local manager software architecture.

\subsubsection{Monitoring and warning function}

The logic of the monitoring function implementation in the local manager is very straightforward. If a sensor message is received from the Serial Port Monitor 
component, the local manager forwards the received sensor message to the web service via the Data-Command Synchronizer component. The received sensor message is also saved in the local manager database. Compared with the web service user interface, the local manager also provides a data representation function for end devices in the local wireless sensor network. Figure 23 shows the local manager user interface which displays the collected sensor data in different curves.

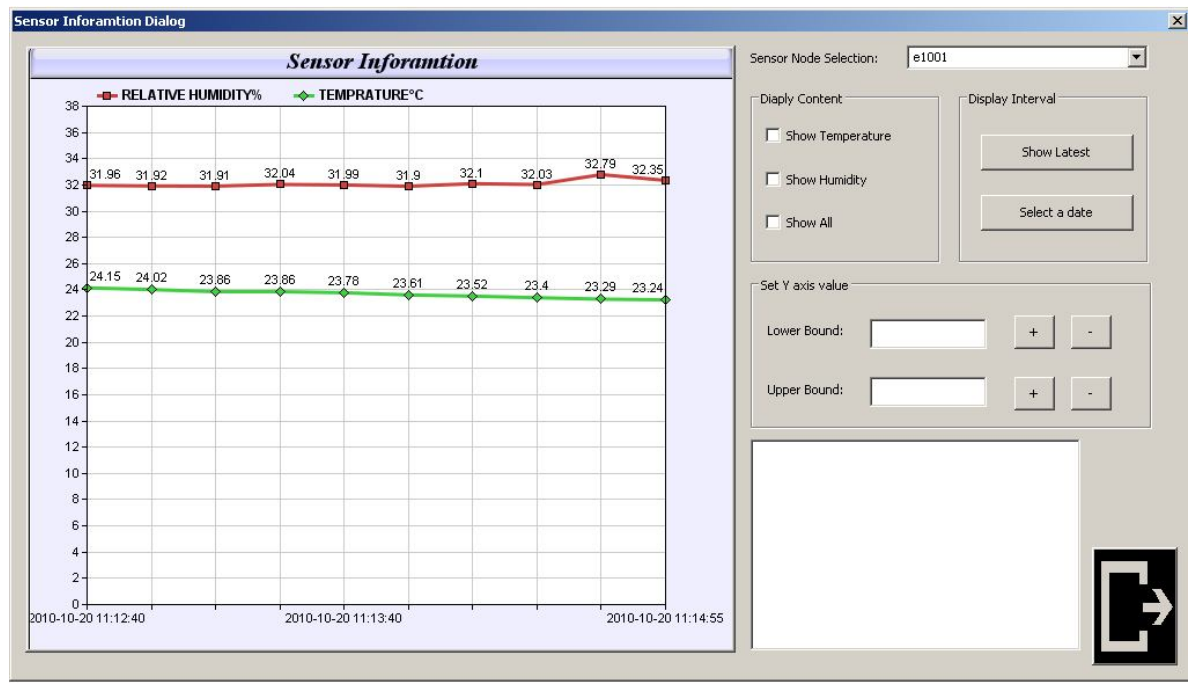

Figure 23. Local manager data monitoring user interface.

By reusing the measured sensor data, a warning function is developed in the local manager. Measured sensor data and wireless device communication status are under surveillance by the local manager. The generated warning messages are sent from the local manager to the web service. Generally, the warning function generates two kinds of warning messages. Firstly, the warning function checks every received sensor value against the sensor threshold. If the sensor value is outside the threshold, a sensor warning message is generated. Secondly, the warning function keeps track of the network status of all wireless devices in the WSN. If the local manager does not receive any status update from a device, after a predefined interval, this device is considered as offline. A device offline warning message is generated. All generated warning messages are forwarded from the local manager to the web service via the 
Data-Command Synchronizer component. The warning function message flow is shown in Figure 24.

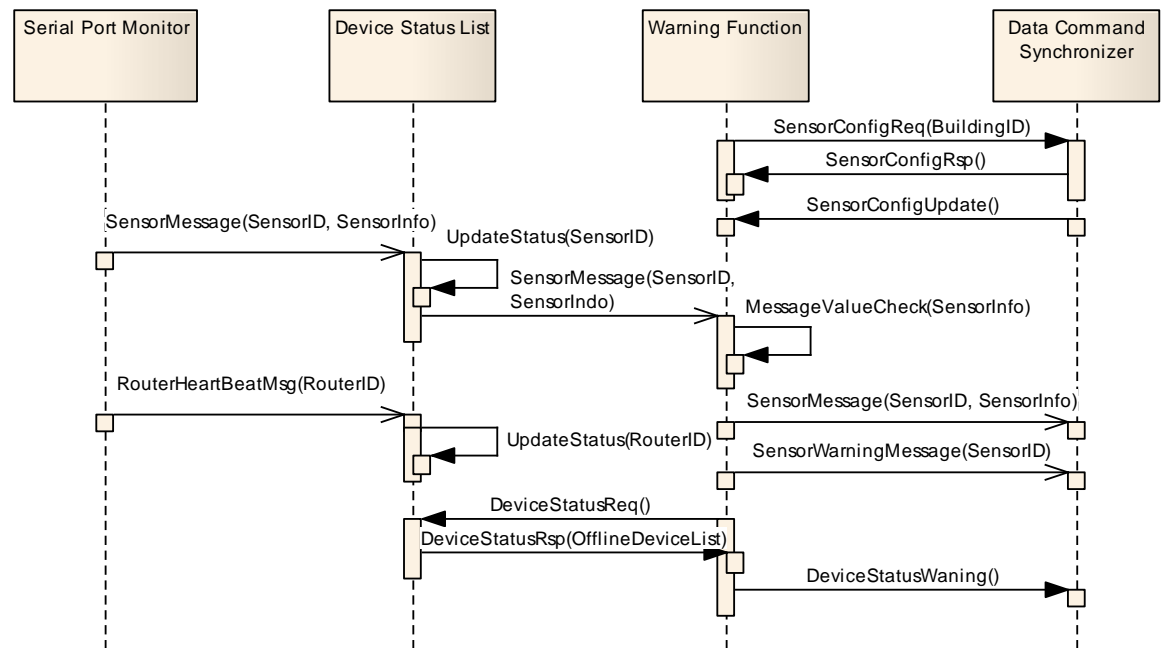

Figure 24. Local manager monitoring and warning function message flow.

During the local manager startup, the warning function updates the end device threshold configuration from the web service. A SensorConfigReq request is sent from the Warning Function module to the web service via the Data Command Synchronizer module. The sensor configuration from web service is reported to the warning function in the SensorConfigRsp message. Additionally, if the sensor configuration is updated during the local manager execution, the sensor configuration update is notified by the Data-Command Synchronizer module via the SensorConfigUpdate command.

If the local manager receives a sensor message via the Serial Port Monitor module, the corresponding end device status is updated in the Device Status List. The sensor message is then checked with the sensor configuration recorded in the warning function. If the sensor information is outside the sensor threshold configuration, the local manager generates a sensor message warning.

The Device Status List records the latest message report time for all devices in the WSN. The end device status is updated every time when the local manager receives the 
sensor message. For routers and controller nodes, the time of the heart-beat message received by the local manager is recorded. The Warning Function module checks the device status by sending DeviceStatusReq request. On receiving this request, the Device Status List module check the device report time against the current timestamp. If the time difference is larger than the predefined interval, the device is considered as offline. The offline device list is sent to the Warning Function module and forwarded to the web service.

\subsubsection{Control function}

The control function is designed aiming at controlling the indoor climate of the occasionally used churches. Different from the city area, around $50 \%$ of the occasionally used churches do not have a stable Internet connection. This requires that the wireless control system can work independent of the web service. For a wireless control system deployed in a cultural building, the local manager contains all logics to manipulate the local system. In order to get the climate setting, the local manager synchronizes data with the web service all the time. The indoor climate control is separated into task control and basic control. In the task control, the indoor climate is adjusted according to the setting during the human activity schedule. During the time when no activity is scheduled, the indoor climate is controlled according to the basic control. The basic control indoor climate configuration is done by the cultural heritage preservation expert. It is the minimum climate requirement for heritage preservation. The major goal for the heritage preservation inside churches is to prevent biological degradation such as mold and insects. In such a case, the temperature and relative humidity are the two most important factors to be manipulated.

In the local manager function implementation, the database is utilized to maintain the controller configurations and relationships. Firstly, the local manager maintains the binding relationship between end devices and wireless control nodes. When an end device is bound with a control node, the control node adjusts the indoor climate according to the sensor information reported from the binding end device. Secondly, the local manager maintains the controller type. The controller type is virtually configured to the wireless control node by the local manager. It can be changed at any time without reprogramming the firmware of the wireless control node. Thirdly, 
different control algorithms are implemented for the wireless control node. Same as the controller type, the control algorithms are also executed and assigned to different wireless control node virtually in the local manager. In order to achieve different control effects, a controller can choose the control algorithm from threshold control, Proportional-Integral-Derivative (PID) control [69] or mold prevention control.

Figure 25 shows the control function database design of the local manager. The control function information is saved in 7 database tables. Table Router List and Sensor List maintain the device information of the wireless sensor network. The Binding Relationship table maintains the relationship between feedback end devices and wireless control nodes. In order to increase the reliability, one controller is always bound with more than one feedback end device. For one wireless control node, the bound end devices are configured with priorities. The Priority field tells the local manager which sensor message feedback should be applied for the wireless control node. The table Controller Type contains a list of all possible controller types in the system. The table Controller Information maintains the configurations of all wireless control nodes. The Router ID field refers to the router index in the Router List table. It states which router is programmed as a wireless control node. The Controller Type field refers to the index of the table Controller Type. It shows the controller type that is connected with the wireless controller. The Controller Status files record the current ON/OFF status of the wireless controller. The status is updated based on the acknowledgement reported from the wireless control node. The Control Algorithm filed indicates the control algorithm configured for the wireless control node. The climate control schedules are saved in table Controller Schedule and Default Schedule. The Controller Schedule table saves all the user issued climate settings for human activities. Such messages are synchronized from the web service by the local manager all the time. The Default Schedule table saves the default configurations for the basic control. It is the climate configuration for heritage preservation purpose. 


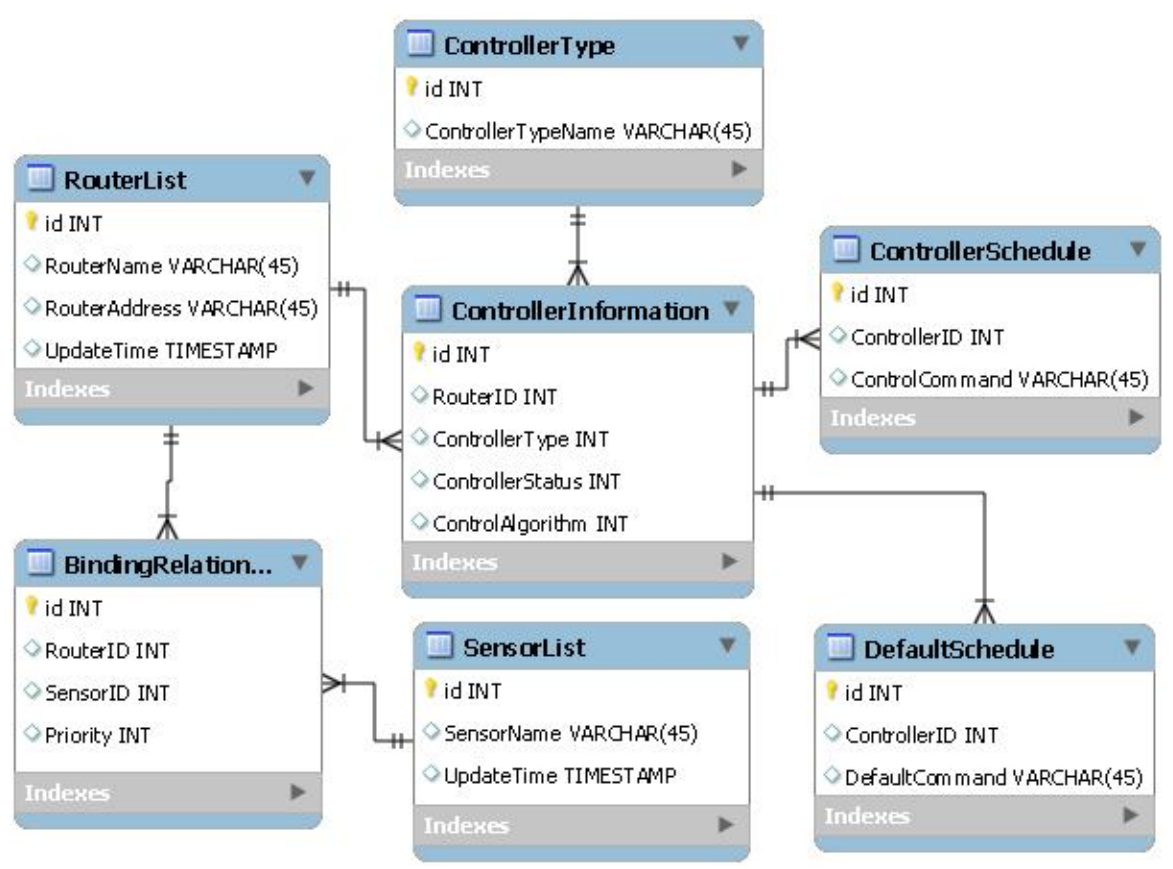

Figure 25. Control function databse design.

As the logic center for a local wireless control system, the local manager generates control commands to each wireless control node. The control command generation process is always triggered by the received sensor message. Once a sensor message is received, the local manager checks the database and gets the bound control node list. According to the binding configuration, more than one wireless controller might bind with one feedback sensor at the same time. Control commands are generated for each wireless control node based on the climate setting, the sensor value and the control logic. The control command creation process is described in the state machine as shown in Figure 26. After start-up, the local manager stays at the IDLE state. Once a sensor message is received, the local manager forwards the message to the web service and jumps to the SYNC state. In this state, the local manager checks the Binding Relationship table to get the binding controller list. If no control node is found, the local manager jumps back to the IDLE state and wait for the next sensor message. Otherwise, the local manager jumps to the CONTROLLR PENDING state. For each bound control node, the local manager generates the specific control command in the CONTROL PROCESS state. When the control command is generated, the local 
manager jumps back to the CONTROLLER PENDING state. If there is no more pending control nodes, the local manager jumps to the IDLE state and waits for the next sensor message.

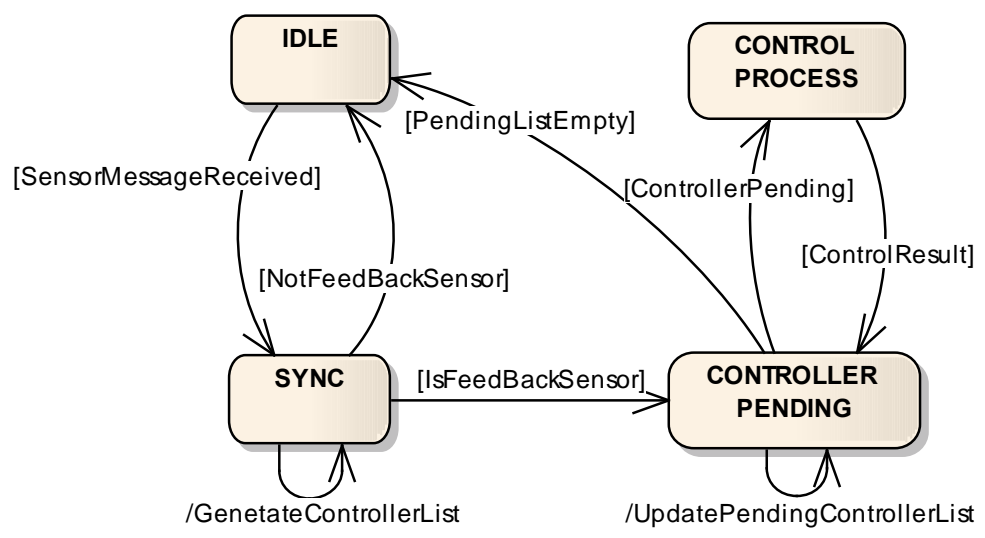

Figure 26. Control command creation state machine.

For each wireless control node, the control command is generated by the local manager in the CONTROL PROCESS state. As shown in Figure 27, when local manager enters this state, the control function component first gets the controller configuration from the table Controller Information according to the device ID. The controller information contains the controller type, the controller status and the controller algorithm. Secondly, the control function component gets the correct schedule from the Controller Schedule table. It contains the current climate setting. After getting all necessary input information, the control function component sends the Get Control Command request to the Controller Algorithm component. Based on the received information, the controller algorithm returns the Control Command with a Turn-on Interval value. When receiving this command, the local manager forwards this command to the corresponding wireless controller node via the concentrator. 


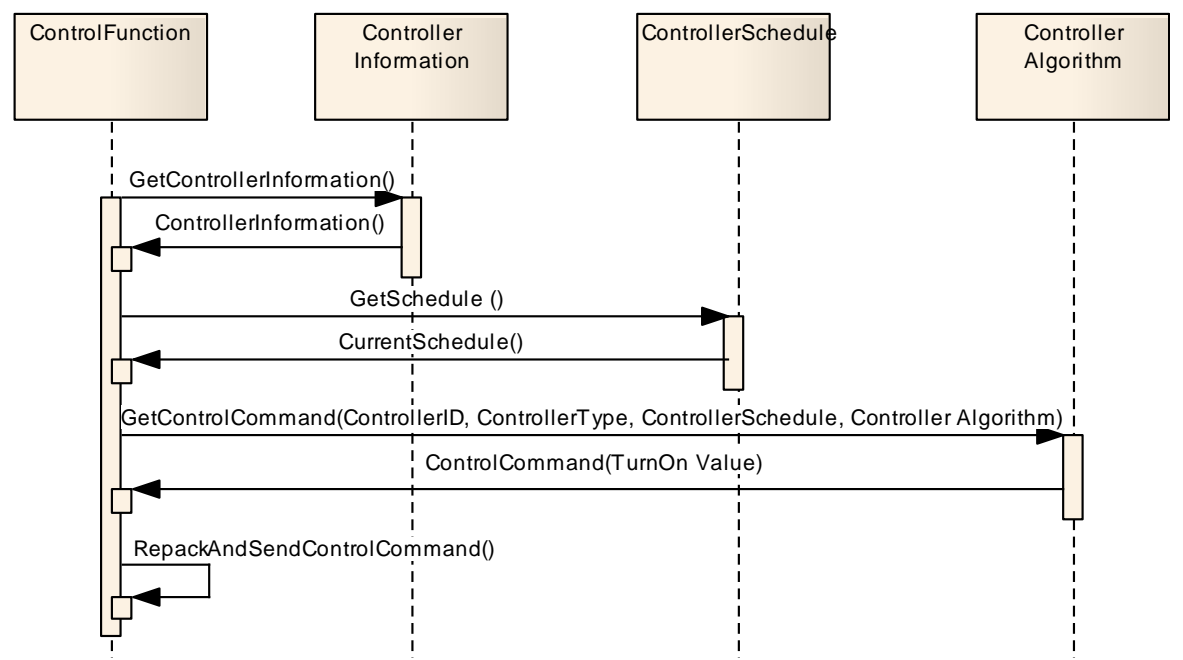

Figure 27. Local manager control function generation message flow.

\subsubsection{Control algorithm implementation}

Three control algorithms are implemented in the local manager control function, which are the threshold control algorithm, mold prevention algorithm and PID algorithm. The threshold algorithm controls the indoor climate within a certain range. This algorithm changes the indoor climate to the configured setting as soon as possible. The mold prevention algorithm calculates the mold risk-free relative humidity level based on the current temperature. This algorithm is mainly utilized for heritage preservation purpose. The PID control algorithm is implemented to minimize the overshoot during the indoor climate control. With careful parameter tuning, the PID algorithm can also eliminate the climate fluctuation which can cause an uncomfortable feeling during human activities. All developed control algorithms can be applied for both temperature control and relative humidity control.

Figure 28 shows an example of temperature threshold control algorithm. The indoor temperature threshold is set between 9 to $11{ }^{\circ} \mathrm{C}$. When the temperature is higher than the upper limit, the radiator is turned off. When the temperature is lower than the lower limit, the radiator is turned on. If the measured temperature is inside the threshold, the radiator keeps the previous operation. For example, if the temperature goes into the threshold from a higher temperature, the radiator is turned off within the threshold. 
Otherwise, the radiator is turned on within the threshold. The purpose of the threshold definition is to prevent the frequently switching on/off of the mechanical power relay. However, large threshold also introduce big temperature change which is not good for the heritage preservation. By default, the threshold is defined as $2{ }^{\circ} \mathrm{C}$. Users can change the configuration according to the specific requirement.

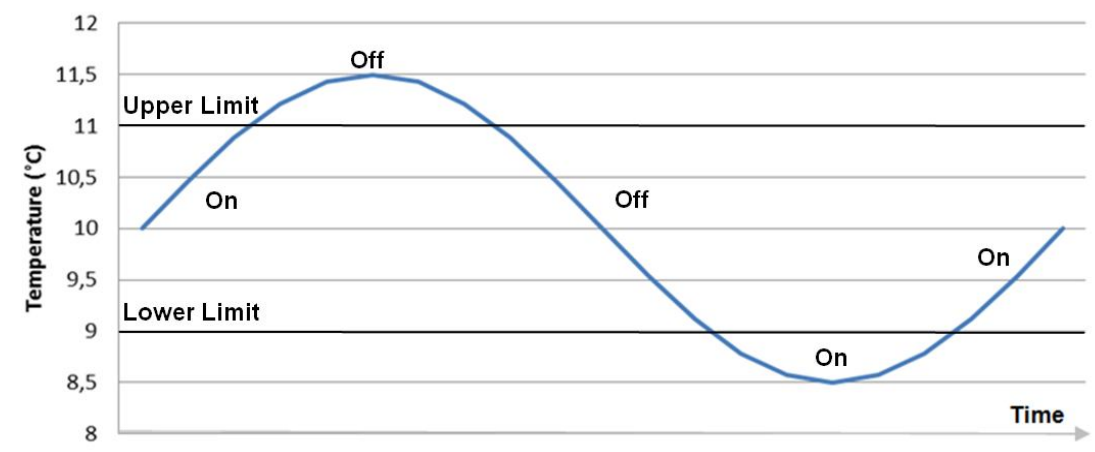

Figure 28. Temperature threshold control algorithm.

A relative humidity control result is shown in Figure 29. In this figure, both the upper and low thresholds are set to $64 \%$. As shown from the curve, the relative humidity jumps between the settings point all the time.

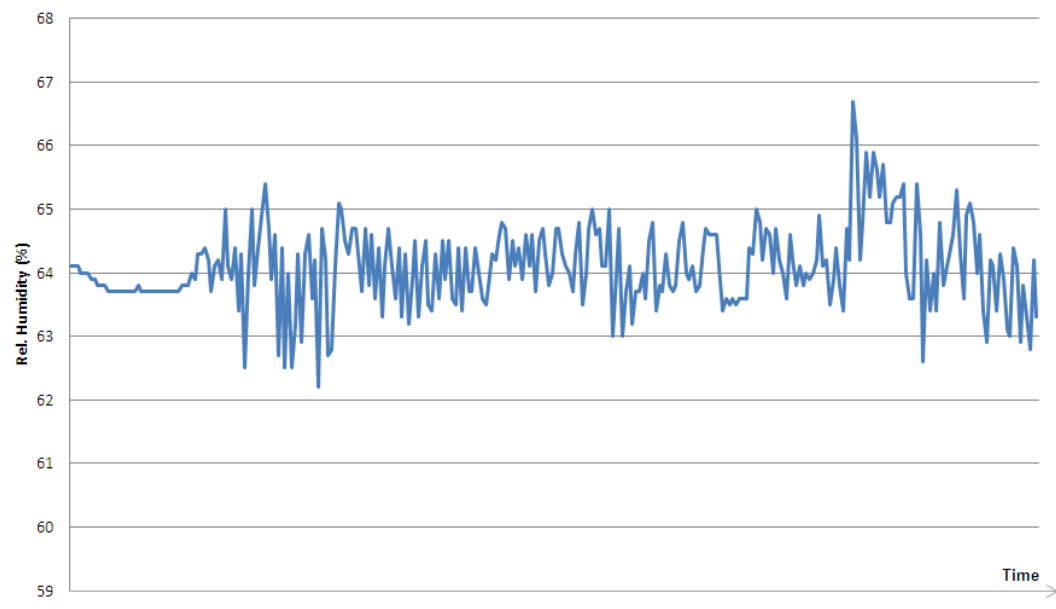

Figure 29. Relative humidity threshold control result. 
Figure 30 shows the mold prevention control algorithm. The mold risk-free relative humidity is calculated based on the current indoor temperature according to Eq.1 [70].

$R H=75+20 \times e^{(-0.1241 \times T)}$

In the mode prevention control algorithm, the target climate setting is dynamically calculated. For a wireless radiator controller, the target temperature is calculated based on the current relative humidity. For a wireless dehumidifier, the target relative humidity is calculated based on the current temperature. The curve indicates the boundary of the mold risk. The red dot indicates the climate with mold risk while the green dots indicate the climates which are mold risk free.

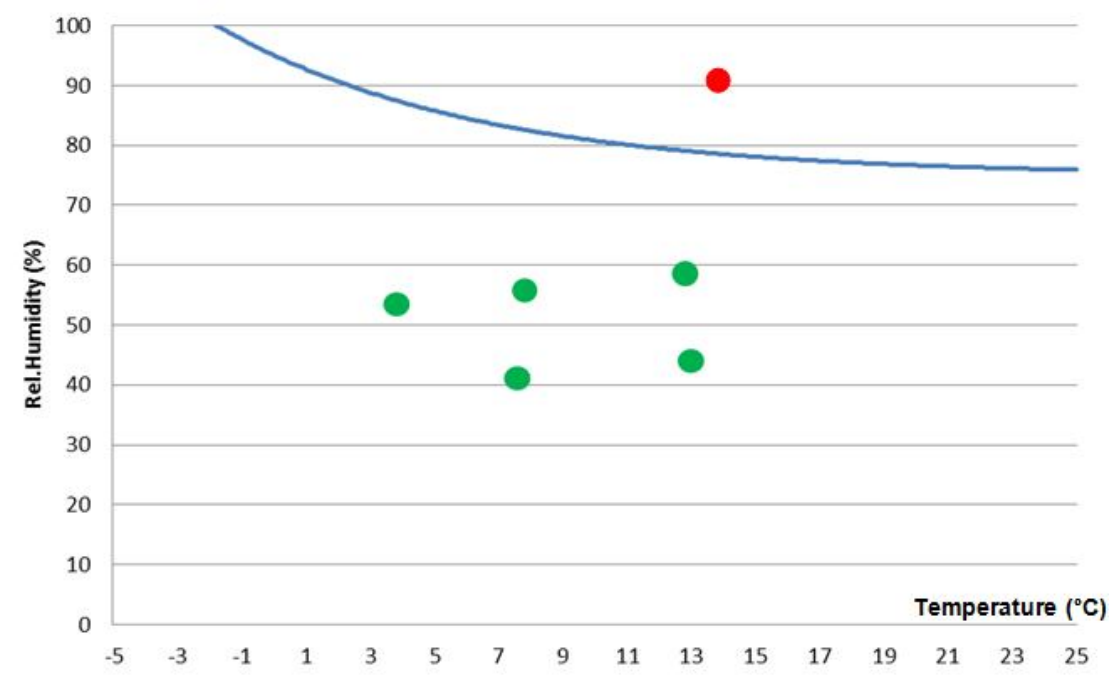

Figure 30. Mold prevention contorl algorithm.

As described in the threshold control algorithm and the mold prevention control algorithm, the wireless control nodes keep working until the feedback sensor value reach the target setting. Such control methods bring climate control overshoots whose amplitude depends on the climate change delay time. Moreover, when the system reaches the target setting, the system stops working until the climate is outside the defined threshold. As a result, the control system generates a lot of climate fluctuation near the setting point. Such fluctuation is not only harmful to the cultural heritage, but 
also, when it is large enough, makes people uncomfortable [71]. In order to reduce the fluctuations, the PID control algorithm, as shown in Eq.2, is implemented in the local manager. In the PID control algorithm, the control device is turned on only for a certain percentage according to the PID calculation. Obviously, the PID control algorithm introduces more power switching into the control device. The solid-state power switch is recommended when utilizing PID control algorithm.

$$
u(t)=K_{p} e(t)+K_{i} \int_{0}^{t} e(\tau) d \tau+K_{d} \frac{d}{d t} e(t)
$$

Where

$\boldsymbol{K}_{\boldsymbol{p}}$ - Proportional gain, a tuning parameter

$\boldsymbol{K}_{\boldsymbol{i}}$ - Integral gain, a tuning parameter

$\boldsymbol{K}_{\boldsymbol{d}}-$ Derivative gain, a tuning parameter

$\boldsymbol{e}$ - Error

$\boldsymbol{t}$ - $\quad$ Time or instantaneous time (the present)

$\tau$ - Variable of integration; takes on values from time 0 to the present $\mathrm{t}$

Figure 31 shows an indoor climate PID control result. The room temperature is configured to $21{ }^{\circ} \mathrm{C}$ between 7:30 AM to 17:00 PM. The rest time the temperature is set to $19{ }^{\circ} \mathrm{C}$. The radiator temperature setting changed from $19{ }^{\circ} \mathrm{C}$ to $21^{\circ} \mathrm{C}$ at time $7: 30$ AM. After 30 minutes, the temperature researched around $20.3{ }^{\circ} \mathrm{C}$. Then, the temperature slowly approached $21{ }^{\circ} \mathrm{C}$ until 17:00 PM. For different control buildings, users can configure the $\mathrm{Kp}, \mathrm{Ki}$ and $\mathrm{Kd}$ value in $\mathrm{Eq} .2$ to reach different control effects.

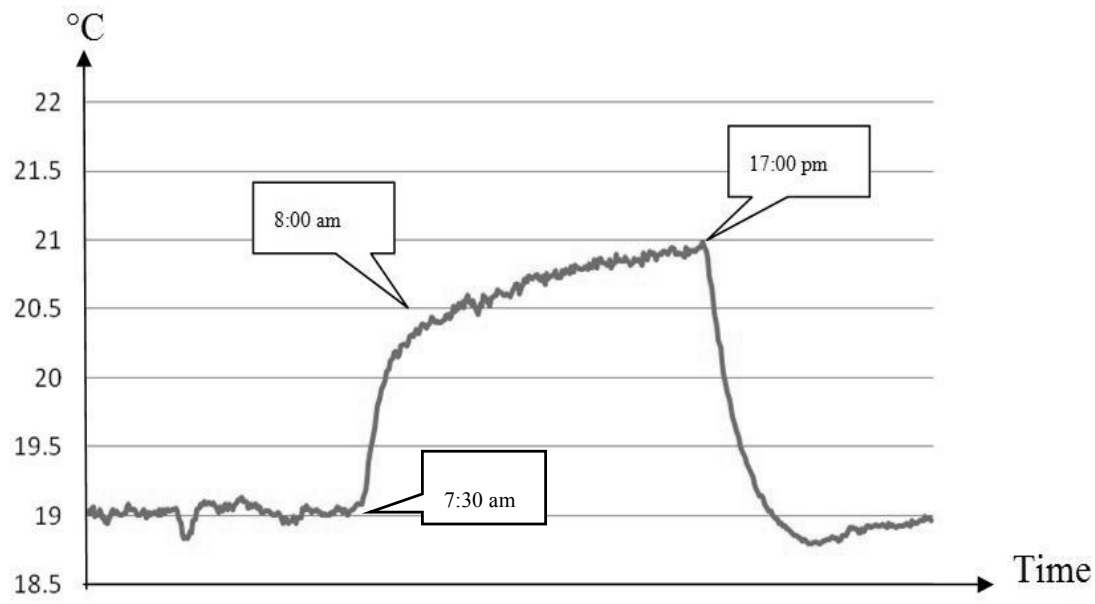

Figure 31. Indoor temperature PID control result. 


\subsection{Web service}

The web service provides web-based user interface for the remote monitoring and control system. The web service also provides interfaces of message uploading and downloading for different local managers. Figure 32 shows the web service architecture. The web-service is developed based on the LAMP (Linux + Apache + MySQL + PHP [72]) server. Since the whole web service is executed passively (execute upon invocation), the CRON service of Linux is utilized to execute time-based schedules, for example, local manager status check. The user interfaces for different functions are developed in Monitoring User Interface (UI), Control UI and Warning UI accordingly. Moreover, a component named "Data Command Synchronizer" is implemented to provide data exchange service for all local managers.

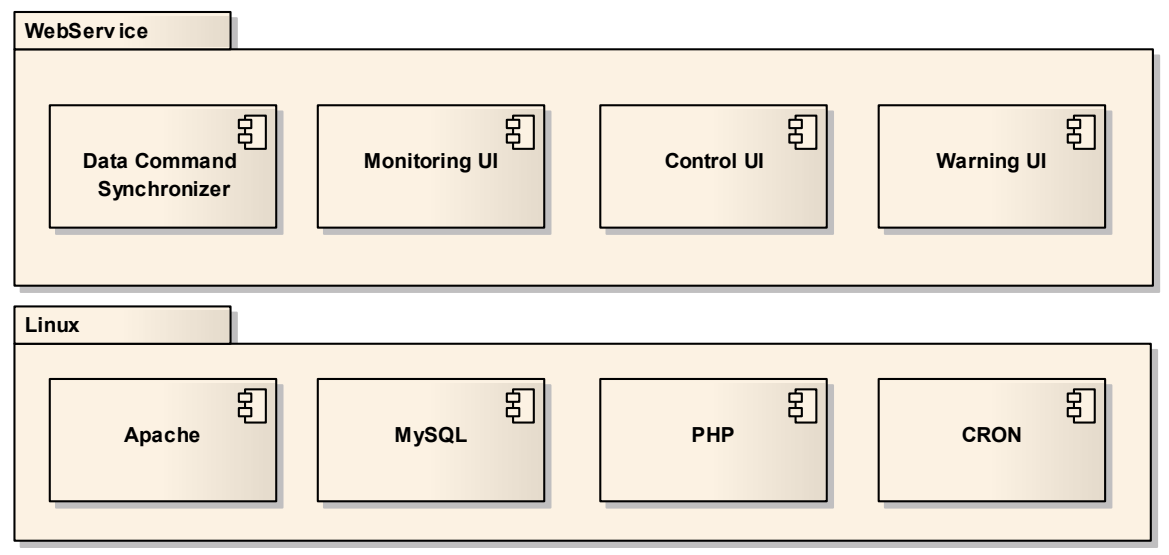

Figure 32. Web service architecture.

The Data Command Synchronizer provides two-way communication interfaces with local managers. The interface can be categorized into "message upload" interfaces, "message download" interfaces and "message update" interfaces. The message upload interfaces allow local managers to upload information to the web service, i.e., received sensor messages and generated warning messages. The message download interfaces allow local managers to download WSN configurations from the web service, for example, wireless control node configuration, sensor interval configuration, climate settings and so on. The message download interfaces are usually invoked during the local manager startup. The message update interfaces forward the web service updating 
to local managers. For example, a sensor threshold configuration is updated in the web service side by the remote user. Such configuration update is notified by this interface to the local manager that the WSN contains the end device.

The user interface of the remote monitoring represents the measured sensor data in curve format. Figure 33 shows the remote monitoring result of one cultural building. The floor plan of the building is shown in the left side. If a building contains more than one floor, users can upload multiple floor plans in the administration pages according to the building construction. The rough location of the end devices are marked in the floor plan. By clicking the end device in the floor plan, the corresponding curve is shown in the diagram to the right. Multiple curves can be shown in the same curve diagram with different colors. The interval of the curve can be changed according to the user configuration. Moreover, the same graph can also be utilized to compare the sensor information from other buildings. 

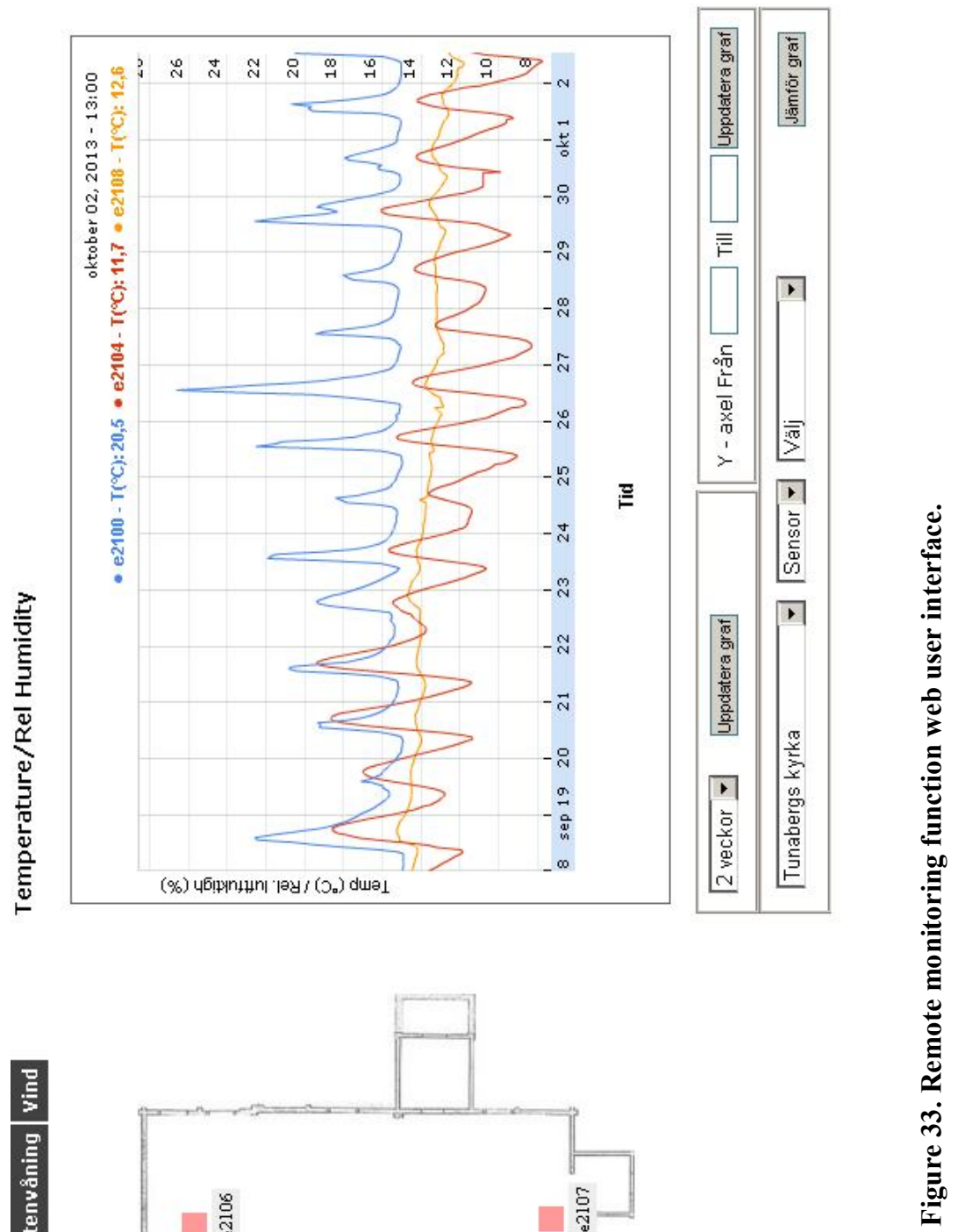
The control function user interface is shown in Figure 34. This page can only be utilized by authorized users. In this page, users can issue climate setting to control the indoor climate in different locations. Figure 34 shows the user interface of adding an activity in a church. The sketch of the church is shown in the left side of the web page. The rough location of the wireless controller is shown in the sketch as well. The configuration interface is shown on the right side. To configure one schedule, users need to select the start date, start time and end time of the schedule. Moreover, users can also configure the command type as daily, weekly, monthly or yearly. The temperature setting is configured in the Set and execute frame according to the controller type. For a radiator, the temperature is configured. For a dehumidifier, the relative humidity is configured.

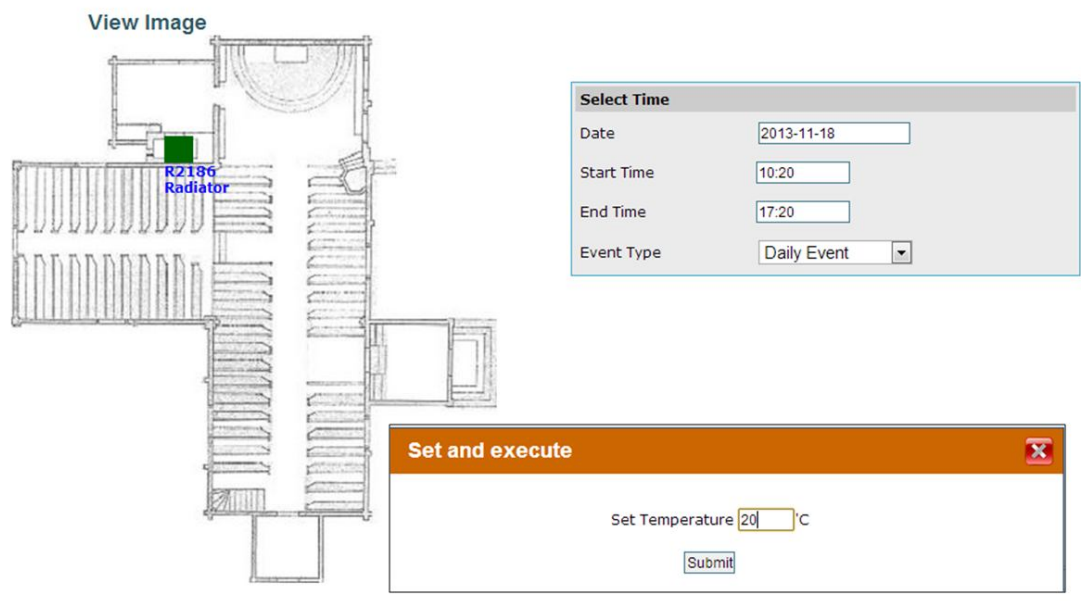

Figure 34. Control function user interface. 


\section{Result}

This dissertation presents a remote wireless monitoring and control solution for cultural heritage preservation purpose. The system consists of three parts, i.e., the wireless sensor network part, the local manager part and the web service part. For each cultural building, a wireless sensor network and a local manager is installed. All local managers communicate with the web service via the Internet.

The ZigBee protocol is utilized in the wireless sensor network part. The performance of the ZigBee network is improved in order to meet the cultural heritage preservation requirements. Specifically, ZigBee routers keep checking the communication link to the concentrator. Once the communication problem detected, the ZigBee router notifies the problem to its child-nodes. On the other hand, the ZigBee end device keeps checking the communication status of its parent device. The end device rejoins the network if the current parent device cannot repair the communication link with the concentrator. During the time when the end device lost the communication with the concentrator, the measured sensor data are buffered in the end device. The buffered senor messages are uploaded to the concentrator when the communication link is reestablished. The wireless sensor network performance improvement is designed in different state machines. In the end device state machine, the battery-driven end device is set to sleep mode as much as possible. An end device powered by a 1.2 Ah battery with 15 minute sensor report interval can work 10 years. The wireless sensor network monitoring and control function are developed based on the ZigBee protocol with improved performance. 
Moreover, a truly battery-driven ZigBee sensor network for wireless monitoring function is developed. An extra microprocessor is utilized as switch to control the power of the ZigBee router. The senor message forwarding process is separated into two steps. In the first step, sensor message reporting from different end devices are scheduled in different time slot. Each end device is assigned with a separated time slot to report the measured sensor data to the router. In the second step, all ZigBee routers wake up at the same time. The concentrator fetches the buffer sensor messages from routers according to the network route. This design eliminates the ZigBee router dependency of the power outlet. The battery-driven router can have at least 1 year battery lifetime when working in a WSN with 50 end devices with 15 minutes sensor message report interval.

The local manager establishes the communication between wireless sensor networks and the web service. In the monitoring function, the local manager forwards the received sensor data to the web service. In the warning function, the web service keeps synchronizing the sensor threshold configuration between the web services. The sensor message warning and device offline warning are generated and forwarded to the web service. The local manager also works as the logic center for the control function. The indoor climate settings are updated from the local manager from the web service. Different control algorithms are implemented in the local manager. The security issues are highly considered in the control function implementation. If the wireless controller lost the communication, no further control can be made before the communication is repaired.

The web service provides user interface for the remote monitoring and control functions. Measured sensor data are presented in curve format. Authorized users could also remote control the indoor climate of different cultural buildings. On the other side, the web service also provides data exchange service with all local managers for message uploading and downloading.

Till today, the developed system has been deployed around 30 places in Sweden and Norway. The system was nominated by the Swedish Embedded Award 2010 and been demonstrated at the Scandinavia Embedded Conference 2010 in Stockholm. 


\section{Summary of the Included Papers}

\section{Paper 1}

Remote Sensing System for Cultural Buildings Utilizing ZigBee Technology by Jingcheng Zhang, Allan Huynh, Qin-Zhong Ye and Shaofang Gong, Proceedings of the $8^{\text {th }}$ International Conference on Computing, Communications and Control Technologies (CCCT 2010), Orlando, USA, pp. 71-77, April 2010.

This paper presents a wireless remote monitoring system using the ZigBee standard. This system is a wireless solution for monitoring purpose in cultural buildings in order to protect cultural heritage. The concept of this system utilizes the ZigBee network protocol to carry and transmit data collected by sensors and store them into both local and remote databases. Thus, users can monitor the measured data locally or remotely. Especially, the power consumption is optimized to extend the lifetime of the batterydriven devices. Moreover, since the system has a modular architecture, it is easy to add extra services into this system.

Author's contribution:

The author proposed the system architecture, designed and implemented the methods described in this work, set-up and performed the test and wrote the article. 


\section{Paper 2}

Design of the Remote Climate Control System for Cultural Buildings Utilizing ZigBee Technology by Jingcheng Zhang, Allan Huynh, Qin-Zhong Ye and Shaofang Gong, Sensors \& Transducers Journal (ISSN 1726-5479), Vol. 118, Issue 7, pp. 13-27, July 2010 .

A wireless solution of remote climate control for cultural buildings is presented in this paper. The system allows users to use web service to control indoor climate in different cultural buildings, like churches. The wireless sensor networks deployed in churches receive the control commands and manage the indoor climate. The whole system is modularly designed, which makes possible an easy service extension, system reconfiguration and modification. This paper includes the system overview and the software design of each part within the system.

\section{Author's contribution:}

The author proposed the system architecture of the remote control system, designed and implemented the message communication of the system and wrote the article.

\section{Paper 3}

Reliability and Latency Enhancements in a ZigBee Remote Sensing System by Jingcheng Zhang, Allan Huynh, Qin-Zhong Ye and Shaofang Gong, Proceedings of $4^{\text {th }}$ International Conference on Sensor Technologies and Applications (SENSORCOMM 2010), Venice/Mestre, Italy, pp. 196-202, July 2010.

This paper presents the methods to improve the reliability and optimize the system latency of the remote sensing system. The concept of this system utilizes the ZigBee network protocol to transmit sensor information and process them at both local and remote databases. The enhancement has been done in different parts in this system. In the ZigBee network part, the network topology is configured and controlled. The latency for message transmitting is also optimized. In the data processing part, the network status check function and data buffer function are introduced to improve the 
system reliability. Additionally, the system latency is measured to compare with the Ad-hoc On Demand Distance Vector (AODV) algorithm used in the ZigBee standard.

Author's contribution:

The author proposed the idea of reliability and latency enhancement in a wireless sensor network, designed and implemented the methods described in this work, set-up and performed the test and wrote the article.

\section{Paper 4}

A Fully Wireless Monitoring and Control System for Protecting Cultural Heritage by Jingcheng Zhang, Allan Huynh, Qin-Zhong Ye and Shaofang Gong, Proceedings of $20^{\text {th }}$ IEEE International Conference on Collaboration Technologies and Infrastructures, Paris, France, pp. 51-57, June 2011.

This paper presents a component-based wireless monitoring and control system. The system is introduced from both the system architecture and function point of view. The paper begins with the introduction of the component design and the communication interaction between them. The system is composed of three components, i.e., the wireless sensor network, the local server and the main server. Wireless sensor networks are deployed in different locations for remote monitoring and control purposes. The monitoring results and control commands are synchronized between the main server and wireless sensor networks via local servers. The test results of the battery lifetime calculation and remote monitoring field test results are presented at the end of the paper.

Author's contribution:

The author proposed and developed the system architecture of a remote monitoring and control system, designed and implemented the methods described in this work, set-up and performed the test and wrote the article. 


\section{Paper 5}

A Communication Reliability Enhancement Framework for the ZigBee Wireless Sensor Network by Jingcheng Zhang, Allan Huynh, Qin-Zhong Ye and Shaofang Gong, Sensors \& Transducers Journal (ISSN 1726-5479), Vol.135, Issue 12, pp. 42-56. 2012.

This paper presents the framework that enhances the communication reliability of wireless sensor network using the ZigBee protocol. The paper begins with the introduction of common communication problems caused by broken links between the sensor module and the message relay, or between different message-relays. Extra message handshake mechanisms are added to solve the mentioned problems. Based on the solution to different problems, a general purpose reliability enhancement framework is developed. The framework contains two state machines for both the sensor module and the massage relay. These two state machines can work together with the ZigBee protocol to enhance the network communication reliability. Moreover, the battery lifetime of the sensor module under link loss is considerably increased after the enhancement.

\section{Author's contribution:}

The author investigated the limitation of ZigBee wireless sensor network, proposed the idea of the reliability enhancement, designed, developed and verified the methods described in this work, set-up and preformed the power consumption test and wrote the article.

\section{Paper 6}

\section{A Web-based Remote Indoor Climate Control System Based on Wireless Sensor}

Network by Jingcheng Zhang, Allan Huynh, Qin-Zhong Ye and Shaofang Gong, International Journal of Sensors and Sensor Networks, Vol. 1, No. 3, June 2013.

This paper presents the design and implementation of a web-based wireless indoor climate control system. The user interface of the system is implemented as a web service. People can login to the website and remotely control the indoor climate of 
different locations. A wireless sensor network is deployed in each location to execute control commands. A gateway is implemented to synchronize the information between the wireless sensor network and the web service. The gateway software also includes scheduling function and different control algorithms to improve the control result. Additionally, the system security and availability are highly considered in this system. The gateway software implements a warning function which sends warning messages when emergency happens. Finally, the whole wireless control system architecture is modularly designed. It is easy to add different control applications or different control algorithms into the system.

Author's contribution:

The author studied the requirement of indoor climate control for cultural heritage buildings, proposed the idea of the wireless remote control system, developed the control system architecture, investigated, developed and compared the effects of different control algorithms on cultural heritage building indoor climate control.

\section{Paper 7}

\section{Design and Implementation of a Truly Battery-Driven ZigBee Wireless Sensor} Network by Jingcheng Zhang, Qin-Zhong Ye, Allan Huynh and Shaofang Gong, Manuscript, September, 2013.

This paper presents the design and implementation of a ZigBee wireless sensor network using battery-driven routers. In order to save the power of the ZigBee router, another microprocessor is utilized as the synchronization controller which controls the power of the ZigBee router according to the wireless sensor network applications. The battery provides power for both ZigBee router and microprocessor. The microprocessor maintains the time of the wireless sensor network applications when the router is powered off. The power is only turned on before the router needs to execute the scheduled application. The wireless sensor network is established by three kinds of wireless devices: the concentrator, the router and the end device. The concentrator configures the wireless sensor network during the network installation. Periodically, the concentrator sends sensor message query to each router in order to collect buffered sensor messages. The router communicates with both end devices and the concentrator. 
The router controls the sensor message report interval of the end device. The router also buffers the sensor messages reported from end devices. For the concentrator, the router uploads buffered sensor messages to the concentrator periodically. The wireless sensor network is optimized from both the collision avoidance and battery lifetime point of view. In order to reduce the message collision, the concentrator allocates a time window for each router. End devices associated with one router are configured to send sensor messages only during the specified time window. Furthermore, the router also controls the sensor report time for each end device. The given time window is separated into many time slots. End devices are configured to report sensor message in different time slots. In order to optimize the router power consumption, an algorithm is developed in the concentrator to determine the sensor message fetching sequence to each router. If a router is not utilized as message relays for other routers, the sensor message from this router is firstly fetched by the concentrator. In this way, the router can go to power off mode right after the message uploading. Additionally, stability enhancement state machines are developed for both router and end device. They help router and end devices to re-establish the communication with the wireless sensor network with battery-powered routers. Finally, the power consumption and battery lifetime is calculated at the end of the paper.

\section{Author's contribution:}

The author proposed the idea, designed and implemented the methods described in this work, set-up and perform the power consumption test and wrote the manuscript. 


\section{BIBLIOGRAPHY}

[1] Atzori L, Iera A. and Morabito G, "SIoT: Giving a Social Structure to the Internet of Things" IEEE Communications Letters, Vol 15, Issue 11, pp. 1931195.

[2] Mandrioli Paolo, Caneva Giulia and Sabbioni Cristina, Cultural Heritage and Aerobiology: Methods and Measurement Techniques for Biodeterioration Monitoring, 2004 Edition, Springer, 2003, pp.47-59.

[3] Drew Gislason, ZigBee Wireless Networking, Newnes, 2008, pp7.

[4] Mailadil T. Sebastian, Dielectric Materials for Wireless Communication, $1^{\text {st }}$ Edition, Elsevier Science, 2008, pp.33-39.

[5] Miyamoto A, Watanabe K and Ikeda K, "Packet loss rate estimation with active and passive measurements" 2012 Asia-Pacific Signal \& Information Processing Association Annual Summit and Conference (APSIPA ASC),

[6] Poul Klenz Larsen, Magnus Wessberg and Tor Broström, "Adaptive ventilation for occasionally used churches" $3^{\text {rd }}$ European Workshop on Cultural Heritage Preservation, Bolzano, Italy, Sep.16-18, 2013.

[7] W. J. Kowalski, "Indoor Mold Growth - Health hazards and remediation", HPAC Engineering, Sep, 2000.

[8] Seth Blumsack, Alisha Fernandez, "Ready or not, here comes the smart grid!" Energy, Volume 37, Issue 1, January 2012, pp. 61-68.

[9] McDaniel, P and McLaughlin, S. "Security and Privacy Challenges in the Smart Grid”, IEEE Security \& Privacy, Volume 7, Issue 3, , pp.75-77, 2009.

[10] V. K. Sood, D. Fischer, J. M. Eklund and T. Brown, "Developing a communication infrastructure for the Smart Grid", 2009 IEEE Electrical Power \& Energy Conference (EPEC), Montreal, pp1-7, 2009. 
[11] A. Zaballos, A. Vallejo and J. M. Selga, "Heterogeneous communication architecture for the smart grid", IEEE Network, Volume 25, Issue 5, pp30-37, 2011.

[12] Luigi Atzori, Antonio Iera and Giacomo Morabitoc, "The Internet of Things: A survey”, Computer Networks, Vol 54, Issue 15, pp2787-2805, 2010.

[13] Friedemann Mattern and Christian Floerkemeier, "From the Internet of Computers to the Internet of Things", LNCS, Vol 6462, pp242-259, 2010.

[14] G. Mulligan, "The internet of things: Here now and coming soon." IEEE Internet Compute, Volumn 14, Issue 1, pp35-36, 2010.

[15] Siok Kheng Tan, Mahesh Sooriyabandara, and Zhong Fan, "M2M Communications in the Smart Grid: Applications, Standards, Enabling Technologies, and Research Challenges", International Journal of Digital Multimedia Broadcasting, Volume 2011, 2011.

[16] Geng Wu, Talwar, S, Johnsson, K and Himayat, N, "M2M: From mobile to embedded internet”, IEEE Communications Magazine, Volume 49 , Issue 4, pp36-43, 2011.

[17] Niyato, D. Lu Xiao and Ping Wang, "Machine-to-machine communications for home energy management system in smart grid", IEEE Communications Magazine, Volume 49, Issue 4, pp53-59, 2011.

[18] S. Kim, S Pakzad, D Culler, J Demmel, G Fenves, S Glaser and M. Turon, "Health monitoring of civil infrastructures using wireless sensor networks", 6th Int. Conf. on Information Processing in Sensor Networks (IPSN), 2007.

[19] K. Chintalapudi, T. Fu, J. Paek, N. Kothari, S. Rangwala, J. Caffrey, R. Govindan, E. Johnson, and S. Masri, "Monitoring civil structures with a wireless sensor network" Internet Computing, Vol 10, Issue 2, 2006. 
[20] J. P. Lynch and K. J. Loh, “A summary review of wireless sensors and sensor networks for structural health monitoring" Shock and Vibration Digest, Mar 2006.

[21] O. Gnawali, K. Jang, J. Paek, M. Vieira, R. Govindan, B. Greenstein, A. Joki, D. Estrin, and E. Kohler, "The Tenet architecture for tiered sensor networks" 4th International conference on Embedded Networked Sensor Systems (SENSYS), 2006.

[22] N. Xu, S. Rangwala, K. Chintalapudi, D. Ganesan, A. Broad, R. Govindan, and D. Estrin, "A wireless sensor network for structural monitoring" 2nd International conference on Embedded Networked Sensor Systems (SENSYS), 2004.

[23] K. Chintalapudi, J. Paek, O. Gnawali, T.S. Fu, K. Dantu, J. Caffrey, R. Govindan, E. Johnson, and S. Masri. "Structural damage detection and localization using netshm". 5th International conference on Information Processing in Sensor Networks (IPSN), 2006.

[24] Matteo Ceriotti1, Luca Mottola1, Gian Pietro Picco, Amy L. Murphy, ,Stefan Guna, Michele Corrà, Matteo Pozzi, Daniele Zonta and Paolo Zanon, "Monitoring Heritage Buildings with Wireless Sensor Networks: The Torre Aquila Deployment", 8th ACM/IEEE International conference on Information Processing in Sensor Networks (IPSN),2009

[25] Abruzzese D, Angelaccio M, Giuliano R and Miccoli L, "Monitoring and vibration risk assessment in cultural heritage via Wireless Sensors Network", 2nd Conference on Human System Interactions (HSI '09), pp586-573, 2009.

[26] Abruzzese D, Angelaccio M, Buttarazzi B and Giuliano R, "Long life monitoring of historical monuments via Wireless Sensors Network", 6th International Symposium on Wireless Communication Systems (ISWCS 2009), pp570-574, 2009. 
[27] Lee, A.M.C. Angeles, C.T. Talampas, M.C.R and Sison, L.G, "MotesArt: Wireless Sensor Network for Monitoring Relative Humidity and Temperature in an Art Gallery", IEEE International Conference on Networking, Sensing and Control (ICNSC 2008). pp1263 - 1268, 2008.

[28] Pahuja Roop , Verma H.K and Uddin Moin, “A Wireless Sensor Network for Greenhouse Climate Control", IEEE Pervasive Computing, Vol 12, Issue 2, pp49-58, 2013.

[29] Ahonen T, Virrankoski R and Elmusrati M, "Greenhouse Monitoring with Wireless Sensor Network", IEEE/ASME International Conference on Mechtronic and Embedded Systems and Applications (MESA 2008), pp403-408, 2008.

[30] Al-Karaki, J.N, Ul-Mustafa, R. and Kamal, A.E. "Data aggregation in wireless sensor networks - exact and approximate algorithms”, 2004. HPSR. 2004 Workshop on High Performance Switching and Routing, Pg 241-245, 2004.

[31] Q. Li, J. Aslam and D. Rus, "Hierarchical power aware routing in sensor networks", Proc. DIMACS Workshop, Pervasive Net, May, 2001.

[32] F. Ye, H. Luo, J. Cheng, S. Lu and L. Zhang, "A two-tier data dissemination model for large-scale wireless sensor networks", Proceedings of the 8th annual international conference on Mobile computing and networking, MobiCom '02, Pages 148-159, 2002.

[33] Milan Lukic, Bogdan Pavkovic, Nathalie Mitton, Ivan Stojmenovic, "Greedy geographic routing algorithms in a real environment", The Fifth International Conference on Mobile Ad-hoc and Sensor Networks, 2009.

[34] Mark A. Perillo and Wendi B. Heinzelman, "Wireless Sensor Network Protocols", online resource, University of Rochester Rochester, NY, USA.

[35] Wei Ye, John Heidemann and Deborah Estrin, "Sensor-MAC (S-MAC): Medium Access Control for Wireless Sensor Networks", 21st International 
Annual Joint Conference of the IEEE Computer and Communications Societies (INFOCOM 2002), New York, NY, USA, June, 2002.

[36] T. van Dam and K. Langendoen, "An adaptive energy-efficient mac protocol for wireless sensor networks", In Proceedings of the First ACM Conference on Embedded Networked Sensor Systems (SenSys), 2003.

[37] G. Lu, B. Krishnamachari, and C. Raghavendra. "An adaptive energy-efficient and low-latency MAC for data gathering in sensor networks", In Proceedings of the Fourth International Workshop on Algorithms for Wireless, Mobile, Ad Hoc and Sensor Networks (WMAN), 2004.

[38] V. Rajendran, K. Obraczka, and J. Garcia-Luna-Aceves, "Energy-efficient, collision-free medium access control for wireless sensor networks", In Proceedings of the First ACM Conference on Embedded Networked Sensor Systems (SenSys), 2003.

[39] M. Perillo and W. Heinzelman, "DAPR: A protocol for wireless sensor networks utilizing an application-based routing cost", In Proceedings of the IEEE Wireless Communications and Networking Conference (WCNC), 2004.

[40] W. Heinzelman, J. Kulik, and H. Balakrishnan, "Adaptive protocols for information dissemination in wireless sensor networks", In Proceedings of the Fifth Annual ACM/IEEE International Conference on Mobile Computing and Networking (MobiCom), 1999.

[41] C. Intanagonwiwat, R. Govindan, and Estrin D, "Directed diffusion: A scalable and robust communication paradigm for sensor networks", In Proceedings of the Sixth Annual International Conference on Mobile Computing and Networks (MobiCom), 2000.

[42] WirelessHART and HART protocol: online resource http://www.hartcomm.org.

[43] ISA100.11a: online resource www.isa.org/isa100. 
[44] C. Perkins, “Ad hoc On-Demand Distance Vector (AODV) Routing”, IETF, RFC3561, 2003.

[45] DD Falconer, "Time division multiple access methods for wireless personal communications", online resource http://www.cs.yale.edu/.

[46] Edsger Dijkstra, Dijkstra's algorithm.

[47] Fiore, Gabriella, Valeria Ercoli, Alf J. Isaksson, Krister Landern, and Maria Domenica Di Benedetto, "Multi-Hop Multi-Channel Scheduling for Wireless Control in WirelessHART Networks" in ETFA, 2009.

[48] Deji Chen, Mark Nixon, Aloysius Mok, WirelessHART Real-Time Network for Industrial Automation, Springer, 2010.

[49] IPv6 over Low-Power WPAN (6lowpan), tools.ietf.org/wg/6lowpan/.

[50] Shelby, Zach, Carsten Bormann, 6LoWPAN: The Wireless Embedded Internet, Willey, 2009.

[51] IETC RFCs, http://tools.ietf.org/html/rfc2894 .

[52] ISO/IEC standard 7498-1:1994.

[53] ZigBee Alliance, "ZigBee and Wireless Radio Frequency Coexistence”, ZigBee Alliance Documents, 2007.

[54] Mihaela Cardei, Resource Management in Wireless Networking (Network Theory and Applications), Springer; 2005 edition, pp265-273.

[55] Institute of Electrical and Electronics Engineers (IEEE 802), Wireless Medium Access Control (MAC) and Physical Layer (PHY) Specifications for Low-Rate Wireless Personal Area Networks (LR-WPANs), IEEE Computer Society, 2004. 
[56] ZigBee Alliance, "ZigBee Application Profiles", ZigBee Alliance Documents, 2007.

[57] Olga Saukh, Efficient Algorithms for Structuring Wireless Sensor Networks, Logos Verlag Berlin, 2009.

[58] Communication Electronics Research Group, www.comelec.itn.liu.se/.

[59] Texas Instrument, www.ti.com.

[60] Allan Huynh, Jingcheng Zhang, Qin-Zhong Ye and Shaofang Gong, "ZigBee Radio with External Power Amplifier and Low-Noise Amplifier", Sensors \& Transducers Journal, Vol 118, Issue 7, 2010.

[61] Allan Huynh, Jingcheng Zhang, Qin-Zhong Ye and Shaofang Gong, "ZigBee Radio with External Low-Noise Amplifier", Sensors \& Transducers Journal, Vol 114, Issue 3, 2010.

[62] Temperature \& Relative Humidity Sensor from Sensirion, http://www.sensirion.com/en/products/humidity-temperature/humidity-sensorsht11/.

[63] John H. Davies, MSP430 Microcontroller Basics, Newnes, Sep, 2008.

[64] Ivan Stojmenovic, Handbook of Sensor Networks: Algorithms and Architectures, Wiley-Interscience, $1^{\text {st }}$ edition, Oct. 2005, pp251-253.

[65] Unknown-Binding, Wood Preservation Manual, International Book Distributors, 1994, pp21-27.

[66] Shahin Farahani, ZigBee Wireless Networks and Transceivers, Newne, Sep. 2008, pp114-118. 
[67] Stefan Björnander, Microsoft Visual C++ Windows Applications by Example: Code and explanation for real-world MFC C++ Applications, Packt Publishing (June 26, 2008).

[68] MySQL database, http://www.mysql.com/.

[69] Guillermo J. Silva, Aniruddha Datta, S. P. Bhattacharyya, PID Controllers for Time Delay Systems, Birkhäuser, 2005.

[70] W. J. Kowalski, "Indoor Mold Growth - Health hazards and remediation", HPAC Engineering, 2000.

[71] Frederrick R, Georgea M, "Thermal comfort during cyclical temperature functions", ASHRAE, 1980.

[72] Jason Gerner, Morgan Owens, Elizabeth Naramore and Matt Warden, Professional LAMP: Linux, Apache, MySQL and PHP5 Web Development, Wrox, 2005

[73] ZigBee Alliance, www.ZigBee.org

[74] WirelessHart, http://www.hartcomm.org

[75] ISA SP100, http://www.isa.org 


\section{Papers}

The articles associated with this thesis have been removed for copyright reasons. For more details about these see:

http://urn.kb.se/resolve?urn=urn:nbn:se:liu:diva-102593 